Vilmos Ágel/Klaus Fischer

\title{
50 Jahre Valenztheorie und Dependenzgrammatik
}

\begin{abstract}
This article celebrates 50 years of dependency grammar and valency theory. The development of both theories is traced historically and an overview of current problems and possible future developments is given. Section 1 poses basic questions of dependency grammar, which derive from the syntactic theory of L. Tesnière: his innovative analysis, developed decades before Chomsky's Syntactic Structures and Greenberg's Universals of Language, not only combines syntactic autonomy with a functional grounding, but also contains a typological perspective. Section 2 sketches a modern version of valency theory, tracing the discussion since Tesnière's Éléments de syntaxe structural and presenting much discussed topics such as the delimitation of complements from adjuncts and multi-dimensional valency models, but also more recent ideas such as typological-structural valency realisation, the interplay of construction and projection in the valency of polysemous verbs, semantic types of valency modification and historical valency change, i.e. the application of valency theory to non-standardised historical varieties. Section 3 addresses definitory problems of a pure dependency grammar, in particular criteria for establishing connexions between words and determining the regent (head), leading to a short comparison of the information contained in dependency and constituency representations. The article does not just take stock but in section 4 also develops ideas for the future development of syntactic theory by introducing constructionist elements into a projectionist framework and by adding the dimension of historical viability to synchronic syntactic theorising.
\end{abstract}

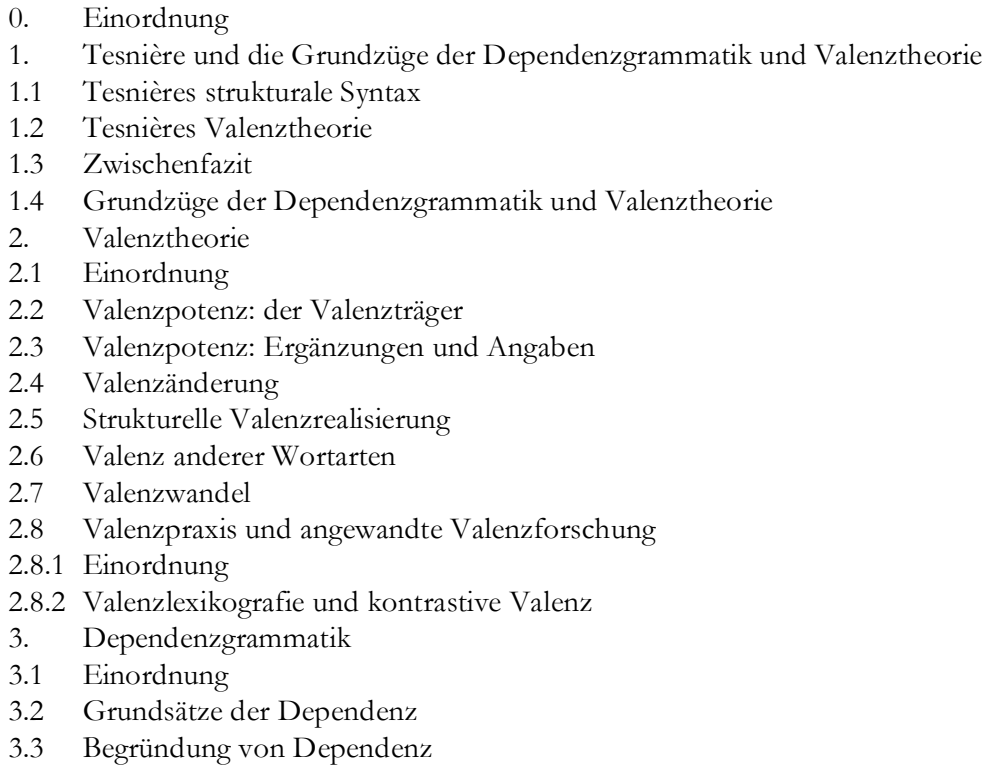


3.4 Dependenz und Konstituenz

4. Quo vadis Grammatiktheorie?

5. Literatur

\section{Einordnung}

Der Begründer der modernen Dependenzgrammatik (DG) und der mit dieser forschungshistorisch stark verbundenen Valenztheorie (VT) ist der Franzose Lucien Tesnière (1893-1954), dessen Hauptwerk „Éléments de syntaxe structurale“ (31976) postum 1959 erschienen ist. Die moderne DG und VT sind somit 50 Jahre alt. Aus diesem Anlass möchten wir Bilanz ziehen. Einschränkend müssen wir jedoch sogleich bemerken, dass hier nur ein äußerst skizzenhafter Überblick dargeboten werden kann. Der Stand der Forschung wird in dem zweibändigen Handbuch „Dependenz und Valenz“ (= Handbuch 2003 und 2006) in insgesamt 121 Artikeln dokumentiert. $^{1}$

Um die nachtesnièresche DG und VT zu positionieren, wird zuerst die größtenteils immer noch aktuelle Syntaxtheorie von Lucien Tesnière vorgestellt und auf aktuelle Forschungsfragen hin überprüft (Kapitel 1). Um den Theoriestand, kritische Punkte in der bisherigen Forschung und Perspektiven für die künftige Forschung zu skizzieren, behandeln wir in den folgenden Kapiteln zuerst die VT (Kapitel 2), dann die DG (Kapitel 3). Wir schließen mit der Frage nach der ,Grammatiktheorie der Zukunft‘ (Kapitel 4).

\section{Tesnière und die Grundzüge der Dependenzgrammatik und Valenztheorie}

\subsection{Tesnières strukturale Syntax}

Die „Éléments“ waren bereits in den 40er-Jahren im Wesentlichen fertig. Ein kurzer Abriss erschien 1953 unter dem Titel „Esquisse d'une syntaxe structurale“. Es existiert eine fast um die Hälfte gekürzte Übersetzung der 670 Seiten umfassenden „Éléments“ ins Deutsche (Tesnière 1980). Erstaunlicherweise gibt es jedoch bis heute keine Übersetzung ins Englische, was die große Diskrepanz zwischen der kontinentaleuropäischen und der angloamerikanischen Rezeption und Verbreitung von DG und VT miterklärt.

Tesnières Theorie der strukturalen Syntax, die erst von seinen Nachfolgern Dependen zgrammatik getauft wurde, hat folgende grundlegende Eigenschaften:

1. Sie ist typologisch-universal angelegt.

1 Dokumentiert wird der Stand der Forschung auch in Ágel/Fischer 2010. Allerdings steht hier entsprechend der Zielsetzung des „Oxford Handbook of Linguistic Analysis“ - der theorievergleichende Aspekt im Vordergrund. Außerdem mussten DG und VT dem mit diesen Theorien weniger vertrauten angelsächsischen Publikum vorgestellt werden. 
2. Sie ist auf die Unendlichkeit der theoretisch produzierbaren oder analysierbaren Sätze hin angelegt.

3. Sie ist autonom.

4. Sie ist funktional.

Tesnière, der selber 20 Sprachen sprach und ca. 60 in den „Éléments“ behandelte, hat großen Wert auf einen Beschreibungsapparat gelegt, der auf beliebige Sprachtypen und Sprachen anwendbar ist. Diesen typologisch-universalen Anspruch versuchte er durch einen modularen Beschreibungsapparat einzulösen. Die Module manifestieren sich in den drei Hauptteilen der „Éléments“, von denen jeder einer der drei Grundrelationen von Tesnières strukturaler Syntax gewidmet ist: connexion, jonction, translation.

Der über 300 Seiten umfassende Konnexionsteil ist der im engeren Sinne dependenzgrammatische Teil des Buches, wo auch die VT als organischer Teil der DG eingeführt wird. Im Konnexionsteil wird die nicht auf dem Prinzip der Binarität von Subjekt (oder NP) und Prädikat (oder VP), sondern auf dem Prinzip der endozentrischen Verbzentriertheit basierende Grundstruktur von Sätzen beschrieben. Endozentrik bedeutet hier, dass sich die Satzstruktur aus dem lexikalischen Verb heraus entfaltet, das Verb also Voraussetzung für die Satzstruktur ist. ${ }^{2}$

Konnexionen betreffen das strukturell induzierte Miteinandervorkommen von Wörtern, Dependenz die hierarchische Ausrichtung der Konnexion. Wir erläutern dies an dem Beispielsatz Peter las sehr gute Bücher, dessen Strukturbaum (stemma) Tesnière wie folgt dargestellt hätte:

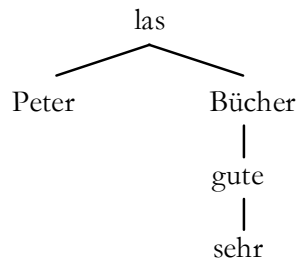

Dieses Stemma erinnert nur äußerlich an einen Konstituenzbaum, es ist ein Dependenz̧baum. Denn die Äste, die die Konnexionen grafisch repräsentieren, stehen nicht für Teil-Ganzes-Beziehungen, sondern - von oben nach unten gelesen - für Rektionsrelationen bzw. - von unten nach oben gelesen - für Abhängigkeitsrelationen. Was oben steht, das Regens, wird nach unten, durch ein oder mehrere Dependentien, strukturell ausbuchstabiert. Strukturell repräsentiert werden soll also, dass es in dem Satz primär um einen spezifischen Akt des Lesens und nicht etwa um eine spezifische Eigenschaft von Peter oder um eine von Büchern geht und

2 Tesnière verwendet den Terminus Endozentrik nicht. Allerdings sind seine für den EndozentrikBegriff relevanten Ausführungen konsequenter als die von Bloomfield (1933: 195), da er davon ausgeht, dass in einem Satz wie lat. filius amat patrem ,der Sohn liebt den Vater' Prädikat und Subjekt nicht einfach amat und filius, sondern ama- und filius...-t sind. Die Verbendung ...-t gehört also nur morphologisch zum Verb, syntaktisch ist sie Teil des Subjekts (Tesnière 1976: 104), s. dazu Abschnitt 2.5.2. 
dass in den Akt des Lesens eine Person als Leser und ein Gegenstand als Gelesenes involviert sind, die als Subjekt und direktes Objekt realisiert werden. Die Substantivformen Peter und Bücher, die strukturell die direkten Dependentien des Regens las sind, könnten auch in ganz andere Akte bzw. Aussagen involviert sein (Bücher sind aufregend, Ich habe Peter lange nicht mehr gesehen). Die grammatischen Funktionen von Peter und Bücher variieren demnach in Abhängigkeit von dem verbalen Regens, aber nicht umgekehrt (Verbzentriertheit).

Das Abhängigkeitsprinzip lässt sich auch auf die Relation von Bücher und gute bzw. von gute und sehr übertragen: Mit gute Bücher ist eine spezifische Eigenschaft von Büchern und nicht eine buchbezogene Gütequalität gemeint. Mit sehr gute ist eine Qualitätsintensivierung und nicht eine Intensivierungsqualität gemeint. Strukturell muss also die Konnexion so beschaffen sein, dass Bücher das Regens des Dependens gute und gute das Regens des Dependens sehr ist. Damit ist Bücher einerseits Dependens von las, andererseits Regens von gute, gute einerseits Dependens von Bücher, andererseits Regens von sehr.

Qua Dependenzstruktur lässt sich der Satz auch konstituentenstrukturell rekonstruieren: Der ganze Satz ist ein Verbalknoten (noud verbal), der aus dem obersten Regens (las) und zwei Substantivknoten besteht. Der eine Substantivknoten (Peter) enthält nur das nominale Regens (das gleichzeitig Dependens des verbalen Regens ist). Der andere Substantivknoten (sebr gute Bücher) enthält das nominale Regens Bücher (das gleichzeitig Dependens des verbalen Regens ist) und den Adjektivknoten sehr gute, der aus dem adjektivischen Regens gute und dem adverbalen Dependens sehr besteht.

Was sich im Gegensatz zu modernen Dependenzgrammatiken jedoch nur teilweise rekonstruieren lässt, ist die Wortstellung. Tesnière unterscheidet streng zwischen der mehrdimensionalen strukturalen Ordnung von Sätzen und der linearen Ordnung, die eindimensional ist und stemmatisch nicht erfasst wird. Bei der Überführung der linearen in die strukturelle Ordnung (Analyse/Rezeption) oder umgekehrt bei der Überführung der strukturellen in die lineare Ordnung (Produktion) hilft jedoch das Wissen um den Sprachtyp (Tesnière 1976: 22ff.). Es gibt zentrifugale Sprachen, die die strukturale Ordnung absteigend - erst das Regens, dann das Dependens - linearisieren (frz. cheval blanc), und umgekehrt zentripetale Sprachen, die die strukturale Ordnung aufsteigend - erst das Dependens, dann das Regens - linearisieren (white horse) > (jap. 白馬; 白, weiß', 馬 ,Pferd $)^{3}{ }^{3}$

Kehren wir aber zurück zur strukturalen Ordnung. Alle Regentien und Dependentien im obigen Beispiel gehören vier Wortkategorien an: Verb, Substantiv,

3 Tesnières Wortfolgetypologie nimmt die von Greenberg (1963) initiierte Wortfolgetypologie in Ansätzen vorweg. 
Adjektiv und Adverb. ${ }^{4}$ Dass es genau diese vier sind, ist kein Zufall. Universal knotenbildend sind nach Tesnière nämlich nur die vier Vollwortarten Verb, Substantiv, Adjektiv und Adverb. Universal sind des Weiteren die Abhängigkeitsrelationen, die die möglichen Konstellationen zwischen den Vollwortarten regulieren: Das Verb ist das oberste Regens, es regiert, kann aber nicht regiert werden. Substantive sind direkte Dependentien von Verben und direkte Regentien von Adjektiven. Adverbien schließlich sind direkte Dependentien von Verben (Er las LANGSAM), Adjektiven (SEHR gute) oder Adverbien (SEHR langsam). Dies führt zur folgenden universalen Dependenzstruktur.

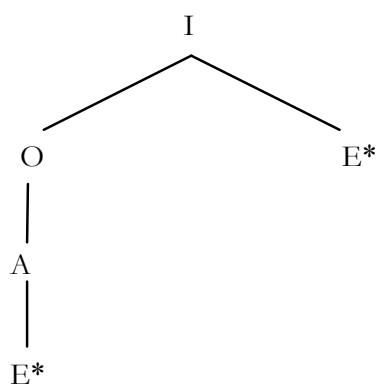

In Anlehnung an Esperanto-Endungen führt Tesnière folgende Symbole ein: $\mathrm{I}=$ Verb; $\mathrm{O}=$ Substantiv; $\mathrm{A}=$ Adjektiv; $\mathrm{E}=$ Adverb. $\mathrm{E}^{*}$ bedeutet eine rekursive Relation, d. h., dass Adverbien auch von Adverbien abhängen können. Ein Symbole enthaltendes Stemma nennt Tesnière virtuelles Stemma, ein Wortformen enthaltendes reales Stemma.

Nun ist unser Einführungsbeispiel im wörtlichen Sinne einfach,

(a) da alle Regentien und Dependentien einfache Wortformen (Peter, las, Bücher, gute, sehr) darstellen,

(b) denen sich dependenziell wohlbestimmte grammatische Funktionen zuordnen lassen: erster Substantivknoten $=$ Subjekt $=$ Peter , Verb $=$ Prädikat $=$ las, , zweiter Substantivknoten $=$ direktes Objekt $=$ sebr gute Bücher, Adjektivknoten $=$ Attribut ersten Grades = sehr gute, Adverbknoten $=$ Attribut zweiten Grades $=$ sebr. ${ }^{5}$

Doch die sprachliche Realität ist wesentlich komplizierter. Beispielsweise bestehen Prädikate oft nicht aus einer einzigen Verbform, sondern aus einem Verb-

4 Tesnières Wortartenbegriffe decken sich nicht mit herkömmlichen Klassifikationen, da sie stärker funktional geprägt sind als sonst üblich. Beispielsweise gehören alle Pronomina und Determinative, die wie das Personalpronomen in Er las sehr gute Bücher kategorial einen Substantivknoten repräsentieren können, zu den Substantiven (mehr dazu in Weber 1997 und Engelen 2003).

5 Tesnière selber benutzt die traditionellen funktionalen Begriffe Prädikat, Subjekt, Objekt und Adverbial nicht, sondern ersetzt diese entsprechend seinem Grundprinzip der endozentrischen Verbzentriertheit durch die Begriffe Zentralnukleus, Aktant und Angabe (s. unten). 
komplex (z. B. haben gelesen). Substantive können nicht nur durch Adjektive, sondern auch durch viele andere Elemente spezifiziert werden (z. B. Lauras). Schließlich können alle Regentien und Dependentien durch Koordination verdoppelt oder vervielfacht werden (z. B. Peter und Pau). Die Grundstruktur von Sätzen lässt sich demnach sowohl durch qualitative als auch durch quantitative Operationen erweitern (Tesnière 1976: 80), z. B. Peter und Paul haben Lauras Buch gelesen:

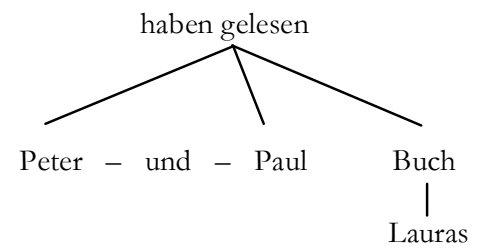

Dieser Satz enthält sowohl einfache als auch komplexe Regentien und Dependentien. Um die Tatsache zu reflektieren, dass Regentien und Dependentien sowohl lexikalische als auch strukturelle (grammatische) Qualitäten haben, spricht Tesnière in Bezug auf einfache und komplexe Regentien und Dependentien von Nuclei (nucléus). Im Falle eines einfachen Nucleus wie las in (1) sind lexikalische und grammatische Merkmale in einer Wortform vereinigt, d. h. - modern gesprochen - las ist sowohl Kern als auch Kopf des Satzes. Dagegen enthalten dissoziierte Nuclei wie haben gelesen oder Lauras ein strukturelles Zentrum (baben bzw. -s), das vor allem grammatische Informationen enthält, und ein lexikalisches Zentrum (gelesen bzw. Laura), das vor allem lexikalische Informationen enthält, d. h. Kern und Kopf sind (relativ) gut isolierbar. Wie man sieht, enthält Tesnières DG alle Elemente einer modernen Phrasenstrukturgrammatik: dem Phrasenbegriff entspricht der Begriff des Knotens. Das strukturelle Zentrum eines Nucleus ist der Kopf, das lexikalische Zentrum der Kern der Phrase. Wenn der Nucleus aus einer Wortform besteht, fallen Kopf und Kern zusammen.

Verantwortlich für die qualitative Erweiterung der Grundstruktur durch komplexe Nuclei ist die Grundrelation der Translation, verantwortlich für die quantitative Erweiterung der Grundstruktur durch Erhöhung der Anzahl der (einfachen wie komplexen) Nuclei ist die Grundrelation der Junktion.

Während Konnexion (Dependenz) die inter-nucleare Relation der Über- und Unterordnung ist, ist Junktion die inter-nucleare Relation der Nebenordnung. Junktion ist ein mit Addition und Multiplikation vergleichbares quantitatives Phänomen (Tesnière 1976: 324), das entweder mit einem Junktiv wie z. B. und (Peter und Paul) oder ohne Junktiv (veni, vidi, vici) operiert.

Wesentlich komplizierter ist die Translation. Da es nur vier kategoriale Typen von Nuclei gibt, deren mögliche Abhängigkeitsrelationen geregelt sind, müssen alle Typen von komplexen Strukturen in diese vier kategorialen Typen überführt werden. Translation ist ein intra-nucleares Relationsgefüge, das aus zwei Typen von 
Operationen besteht (Tesnière 1976: 364): (1) Kategorienwechsel und - als dessen Folge - (2) Funktionswechsel.

Unsere Beispiele für Translation sind die komplexen Nuclei Lauras und haben gelesen. Da der Nucleus Lauras in dem Substantivknoten Lauras Buch als Dependens des Substantivs Buch vorkommt, muss er im Sinne der universalen Dependenzstruktur kategorial ein Adjektiv sein. Ähnlich muss der Nucleus haben gelesen kategorial ein Verb sein. Da jedoch weder Lauras zugleich zwei Wortarten (Substantiv und Adjektiv) noch ein komplexer Nucleus, der aus mindestens zwei Wörtern besteht, einer einzigen Wortart angehören kann, kann es sich bei den Kategorien von Lauras und haben gelesen unmöglich um die genuinen Wortarten Adjektiv bzw. Verb, sondern nur um operativ erzeugte Kategorien Adjektiv bzw. Verb handeln. Die Translationstheorie beschreibt die Mechanismen und die Typen von solchen kategorialen Erzeugungsprozessen.

Jede Translation enthält einen Transferenden, ein Wort, dessen Kategorie verändert werden soll, und einen Translator, der ein freies, gebundenes oder NullMorphem ist, das die kategoriale Überführung grammatisch bewirkt. Das Ergebnis ist das Translat, also der operativ erzeugte Nucleus: Das Flexiv $-s$ überführt das Substantiv Laura in den adjektivalen Nucleus Lauras $(\mathrm{O}>\mathrm{A})$, der als Resultat der Translation strukturell Dependens und funktional Attribut des Substantivs Buch sein kann. Im Falle des Translats haben gelesen wurde der verbale Transferend lesen mithilfe des auxiliaren Translators baben aus einer verbalen Subkategorie, die wir heute eine synthetische Verbform nennen würden, in eine andere, eine analytische Verbform, überführt, also I > I (Verb translatiert zu Verb). ${ }^{6}$

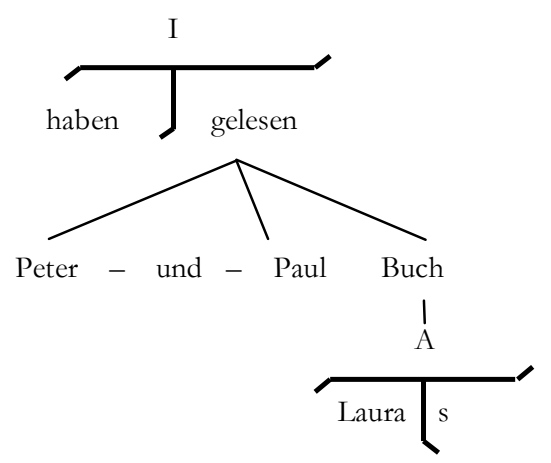

Den Abschnitt abschließend möchten wir nun auf die anfangs erwähnten vier grundlegenden Eigenschaften von Tesnières DG zurückkommen. Dass sie

1. typologisch-universal und

6 Zur grafischen Darstellung der Translation wählt Tesnière sinngemäß ein T. Oberhalb des Querbalkens steht das Translat (oder dessen Symbol), unterhalb des Querbalkens stehen links und rechts des vertikalen Strichs des T Transferend und Translator oder umgekehrt. 
2. auf die Unendlichkeit der theoretisch produzierbaren oder analysierbaren Sätze hin angelegt ist,

hat die obige Skizze der Grundzüge der Theorie hoffentlich überzeugend gezeigt. Was ist aber darunter zu verstehen, dass die strukturale Syntax

3. autonom und

4. funktional ist?

Unter Autonomie seiner Syntax versteht Tesnière einerseits, dass die Syntax, die die innere Form des Satzes untersucht, wohlunterschieden von der Morphologie ist, die die äußere Form des Satzes untersucht (Tesnière 1976: 34). Die Syntax hat ihre eigenen Gesetze.

Autonom ist die Syntax andererseits, weil sie sich ebenso deutlich von der Semantik unterscheidet wie von der Morphologie (Tesnière 1976: 40ff.). An diesem Punkt nimmt Tesnière die Argumentation des frühen Chomsky vorweg. Der beste Beweis für die Unabhängigkeit von Syntax und Semantik sind nämlich nach Tesnière (1976: 40f.) semantisch absurde Sätze wie Rückwärtiges Schweigen verstimmt zulässigen Schleier (le silence vertébral indispose la voile licite), die strukturell korrekt sind und dieselbe Struktur aufweisen wie sinnvolle Sätze.

Die Autonomie der Syntax gegenüber der Semantik bedeutet allerdings für Tesnière nicht, dass die Semantik ein linguistisch uninteressanter Gegenstand wäre, ganz im Gegenteil: Die Bedeutung ist letzten Endes der Daseinsgrund der Struktur und insofern indirekter Gegenstand der Syntax (Tesnière 1976: 40).

Dass Tesnières Syntaxtheorie funktional ist, haben wir an der Konnexionstheorie und deren Zusammenwirken mit der Translationstheorie gesehen. Die einfachen Nuclei beim Einführungsbeispiel (1) ließen eine direkte funktionale Interpretation der Nuclei und Knoten zu. Damit die funktionale Interpretation auch bei Sätzen mit komplexen Nuclei wie in (3) gewährleistet wird, wurden neben genuinen auch durch Translation erzeugte Nucleuskategorien zugelassen. Denn Struktur gibt es nach Tesnière nur in dem Maße, in dem es Funktionen gibt, woraus folgt, dass die strukturale Syntax gleichzeitig eine funktionale Syntax ist (Tesnière 1976: 39).

Wir interpretieren Tesnières Funktionsauffassung als einen konsequenten Versuch, die autonome grammatische Struktur durch eine theoretische Verbindung der semantischen Ebene mit der der grammatischen Funktionen zu motivieren.

\subsection{Tesnières Valenztheorie}

In seiner berühmten Drama-Metapher vergleicht Tesnière (1976: 102) den Satz mit einem kleinen Drama. So wie ein Drama ein Geschehen, Akteure und (zeitliche, räumliche usw.) Umstände umfasst, enthält der Verbalknoten einen Zentralnucleus, der ja gemäß der universellen Dependenzstruktur zwei Typen von Knoten regieren kann: Substantivknoten und Adverbknoten: 
(4)

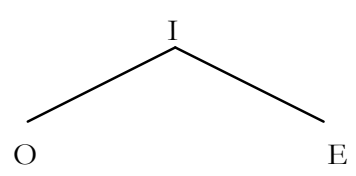

(5)

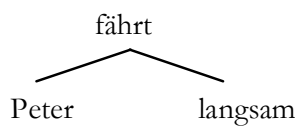

Dem Drama-Geschehen entspricht der Zentralnucleus, der ein (einfaches oder translatiertes) Verb ist (fährt) und den man in der modernen VT verbalen Valenzträger nennt. Die funktionale Entsprechung der Drama-Akteuere sind die Aktanten, die Substantivknoten darstellen (Peter) und modern auch Ergänzungen oder Komplemente genannt werden. Die funktionale Entsprechung der Umstände sind die Circumstanten, die Adverbknoten darstellen (langsam) und auch Angaben oder Supplemente genannt werden.

Das traditionelle Prinzip der Binarität von Subjekt (oder NP) und Prädikat (oder VP) wird also durch Verbzentriertheit ersetzt, die eine funktionale Dreiteilung des Satzes - verbaler Valenzträger, Aktant, Angabe - begründet, die kategorial Verb(äquivalent), Substantiv(äquivalent), Adverb(äquivalent) - abgesichert ist.

Allerdings sind weder Akteure und Umstände noch Aktanten und Angaben gleichwertige Partizipanten eines Dramas bzw. Satzes. Konstitutiv für ein Drama sind Geschehen und Akteure, konstitutiv für Sätze der Zentralnucleus und die Aktanten. Demgegenüber haben die Umstände im Drama bzw. die Angaben im Satz eine externe, die konstitutiven Partizipanten rahmende Funktion. Zwischen I und E besteht nur eine Dependenzrelation, zwischen I und O besteht zusätzlich zur Dependenz- auch eine Valenzrelation. Um diese einzuführen, benutzt Tesnière einen anderen berühmten Vergleich, die Atom-Metapher. Nach dieser kann man „das Verb mit einem Atom vergleichen, an dem Häkchen angebracht sind, so daß es - je nach der Anzahl der Häkchen - eine wechselnde Zahl von Aktanten an sich ziehen und in Abhängigkeit halten kann. Die Anzahl der Häkchen, die ein Verb aufweist, und dementsprechend die Anzahl der Aktanten, die es regieren kann, ergibt das, was man die Valenz des Verbs nennt.“ (Tesnière 1980: 161).

Valenz ist also nach Tesnière die Anzahl der potenziellen, in der Bedeutung des Verbs verankerten Aktanten eines verbalen Valenzträgers. Beispielsweise fordert frz. donner oder engl. to give drei Aktanten. Diese Verben sind also unabhängig davon dreiwertig, dass es neben Sätzen wie Alfred donne le livre à Charles, Alfred gibt Karl das Buch' (Tesnière 1976:107) auch welche wie Alfred donne aux pauvres (,Alfred gibt den Armen') oder Alfred donne la main (,Alfred hält die Hand') gibt, in denen die Valenzpotenz von donner jeweils nur teilweise realisiert wurde (Tesnière 1976: 239).

Tesnière unterscheidet drei Arten von Aktanten (Tesnière 1976: 107ff.). Gemeinsam ist diesen einerseits die dependenzgrammatische Form: Sie sind Substantiv- 
knoten. Gemeinsam ist ihnen andererseits die Propositionsbildung mit dem verbalen Nukleus. Die Aktanten unterscheiden sich dagegen relational-semantisch: Der Erstaktant (traditionell: Subjekt) im Aktivsatz ist der Aktant, der eine Tätigkeit ausführt, der Zweitaktant der, dem eine Tätigkeit/Handlung widerfährt, der Drittaktant der, zu dessen Nutzen oder Schaden etwas geschieht. Man erkennt hier unschwer die späteren Tiefenkasus bzw. semantischen oder Theta-Rollen.

Tesnière, der entsprechend seinen sprachtypologischen Interessen und Kenntnissen die einzelnen formalen Typen von Aktantenrealisierungen in verschiedenen Sprachen Revue passieren lässt (Tesnière 1976: 111ff.), entgeht nicht das auch für die moderne Valenztheorie zentrale Problem der Unterscheidung von Ergänzungen und Angaben. Besonders problematisch sind dabei die Präpositionen, die angesichts der universalen Dependenzstruktur Translatoren sowohl für substantivische als auch für adverbiale Translate sein müssen. Beispielsweise ist in dem Satz Alfred donne le livre à Charles à Paris die Kette à Charles ein Substantiväquivalent, während die Kette à Paris ein Adverbäquivalent ist:

(6)

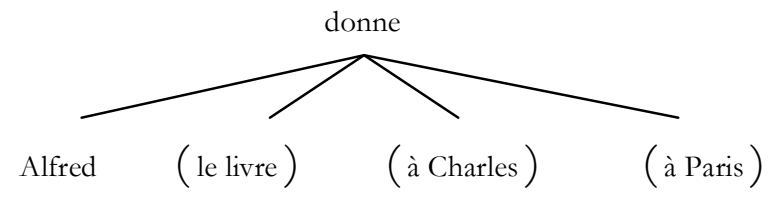

Hier muss mit Hilfe von von der Dependenzstruktur unabhängigen Valenzkriterien entschieden werden, warum à Charles eine Translation von $\mathrm{O}$ (Charles) $>\mathrm{O}(\grave{a}$ Charles) darstellt, während à Paris eine Translation von $\mathrm{O}$ (Paris) $>\mathrm{E}$ (à Paris) ist:

(6a)

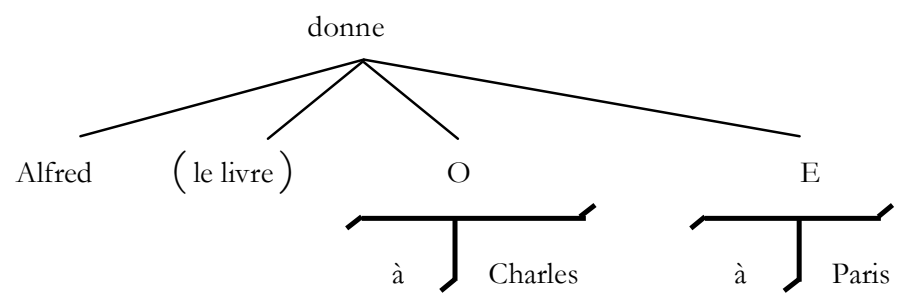

Tesnière kann hier semantische Kriterien (Propositionsbildung, semantische Rolle) und das Kriterium der grundsätzlichen Fakultativität der Angaben heranziehen. Außerdem kann die Kette à Paris durch ein einfaches Adverb (ici, la ) ersetzt werden, aber nicht die Kette à Charles. Als typologisches Argument kommt noch dazu, dass der Kette à Charles in den Kasussprachen ein einfaches Substantiv im Dativ entspricht (Tesnière 1976: 114f.). 


\subsection{Zwischenfazit}

Tesnière zeigt nicht nur, dass eine Syntaxtheorie ohne VT nicht möglich ist, er leitet seine VT auch sehr konsequent aus seiner Syntaxtheorie ab. Umgekehrt zeigt er, dass Valenz eine zusätzliche Motivierung der universalen Dependenzstruktur darstellt, indem die Valenzrelation auf der Rektion von I zu O operiert.

In Tesnières Werk kommen alle Grundfragen von modernen VTn ausführlich und sehr instruktiv zu Wort: (a) die Frage nach dem Valenzträger; (b) die Frage nach der Unterscheidung bzw. Unterscheidbarkeit zwischen Ergänzungen und Angaben; (c) die Unterscheidung zwischen Valenz und deren obligatorischer oder fakultativer Realisierung und (d) das Problem der strukturellen Valenzrealisierung, also die typologische Frage, inwieweit die Struktur einer Einzelsprache die Formen und Typen möglicher Aktanten beeinflusst.

$\mathrm{Da}$ in Sprachen wie dem Englischen oder Chinesischen Aktanten nicht nur morphologisch oder präpositional, sondern auch positional markiert werden, betrifft das Problem der strukturellen Valenzrealisierung auch eine prinzipielle Frage der DG, nämlich die, ob es sinnvoll ist, Tesnières rigide Trennung zwischen strukturaler und linearer Ordnung aufrechtzuerhalten. Unter den modernen gibt es auch projektive, d. h. wortstellungserhaltende, Dependenzgrammatiken (s. Eroms/ Heringer 2003). Dabei gilt Position als der morphologischen Kennzeichnung von Ergänzungen äquivalentes syntaktisches Mittel (Fischer 1997).

\subsection{Grundzüge der Dependenzgrammatik und Valenztheorie}

Gemeinsam allen Grammatiktheorien ist es, dass sie davon ausgehen, dass sich hinter der zeitlichen Abfolge gesprochener Zeichen oder der räumlichen Anordnung geschriebener Zeichen mehrdimensionale Strukturen verbergen, deren Architektur sich nicht einfach an den Daten ablesen lässt. Für die Darstellung dieser Architektur muss die Syntaxtheorie eigene Kategorien und Relationen bereitstellen. Das dependenzgrammatische Autonomieprinzip à la Tesnière basiert auf der kategorialen Unabhängigkeit der syntaktischen Theoriekomponente. Dabei werden semantische (und pragmatische) Differenzen und Oppositionen syntaktisch reflektiert, wenn diese auf syntaktische Kategorien abgebildet sind, aber die Syntax wird nicht auf den Ausdruck von Semantik (und Pragmatik) reduziert. Die Nichtreduzierbarkeit ergibt sich nicht nur daraus, dass nicht jede semantische (und pragmatische) Differenz und Opposition auf syntaktische Kategorien abbildbar ist, sondern auch aus den Mechanismen des Sprachwandels und der Grammatikalisierung. Die DG und insbesondere die VT legen großen Wert nicht nur auf die typologische, sondern auch auf die sprachbistorische und variationslinguistische Adäquatheit der Theoriebildung. Die Theorie soll nicht nur auf einige wenige moderne Schriftsprachen, sondern auf beliebige Sprachen, Vollvarietäten und Sprachstufen anwendbar sein. 
Die Unterdeterminierung der Satzstruktur durch die sprachlichen Formen ist einer der Gründe, warum es unterschiedliche grammatische Theorien gibt. Damit ist gemeint, dass die Satzstruktur nicht vollständig durch sprachliche Zeichen determiniert ist, wie es z. B. in logischen Kunstsprachen der Fall ist. Deshalb gibt es Raum für konkurrierende Hypothesen und deshalb können die Begrifflichkeiten in den verschiedenen Grammatiktheorien nicht eins-zu-eins einander zugeordnet werden. Das sind keine bloß Notations-, sondern substantielle Unterschiede.

Ein zweiter Grund, warum es unterschiedliche grammatische Theorien gibt, ist, dass keine Einigkeit darüber besteht, welche Begriffe für eine Beschreibung der Sprachstruktur die grundlegenden (z. B. Dependenz oder Konstituenz) bzw. überhaupt notwendig sind (z. B. funktionale Begriffe wie Subjekt und/oder formale wie NP). Ein dritter, welcher Aufwand bei der Beschreibung der Struktur betrieben werden soll bzw. wie abstrakt die Strukturbeschreibungen sein dürfen, um eine methodisch gesicherte Verbindung zwischen Theorie und Empirie aufrecht zu erhalten. Dieser Punkt ist eng verbunden mit der Frage, welche Perspektive eine Theorie bei der Beschreibung und Erklärung von grammatischen Konstruktionen einnehmen soll:

(a) eine projektionistische (von den Elementen auf das Ganze) oder eine

(b) konstruktionistische (umgekehrt).

Die DG ist eine im Kernbereich projektionistische Theorie, sie stellt die Struktur von Einzellexemen ausgehend dar: sie ist primär eine Wortgrammatik. Einzellexeme haben ein Kombinationspotenzial bzw. eine Kombinationstoleranz, die die Strukturen stiftet, nicht verhindert oder eben ausschließt (Konnexions-, Junktion-, Translations- und Valenzpotenzial). Die DG ist also - in dieser Beziehung ähnlich etwa der HPSG - keine „Einsetztheorie“ (Feilke 2004: 54), für deren lexikonunabhängige syntaktische Strukturen das Lexikon lediglich die „Füllung“ liefert. Ein bedeutender Teil der Grammatik ist im Lexikon - im Konnexions-, Junktions-, Translations- und Valenzpotenzial von Einzellexemen - abgelegt.

Die wortgrammatische Perspektive der DG hat eine Reihe von Konsequenzen:

1. Das Kombinationspotenzial von Einzellexemen beruht auf ihrer Bedeutung und ihrem syntaktischen Potenzial. Im Sinne des dependenzgrammatischen Autonomieprinzips können also Dependenzäste meist direkt semantisch interpretiert werden. Aber es wird keine Identität oder Eins-zu-Eins-Entsprechung der strukturellen mit der semantischen Ebene angenommen, wie z. B. von der Kategorialgrammatik oder gewissen funktionalen Ansätzen. Vielmehr setzt die DG als strukturalistische Theorie eine unabhängige Strukturebene an. Nur so kann der Grad der Entsprechung syntaktischer und semantischer Strukturen überhaupt festgestellt werden (Welke 1988, Helbig 1992). Der wortgrammatische Ausgangspunkt ist gewählt, um die Unabhängigkeit syntaktischer Strukturen möglichst gering zu halten und so weit wie möglich semantisch zu motivieren. Diese Zielsetzung rückt die DG wiederum in die Nähe der Kategorialgrammatik und funktionaler Theorien. 
2. Der Ausgangspunkt von Einzellexemen macht sowohl eine Interpretation der DG als Rezeptionsgrammatik als auch als Produktionsgrammatik möglich. Ersteres, weil das Hörerwissen um das durch Framing und Priming gestützte syntaktisch-semantische Potenzial von Einzellexemen zu verlässlichen Analysen führt (Heringer 1984 und 1985, Gansel 2003). Letzteres, weil die Produktion von Äußerungen von der Wahl einzelner Lexeme auf der Grundlage der Kommunikationsintention und - trivial, jedoch entscheidend - den Möglichkeiten des im Wortschatz der jeweiligen Sprache verankerten konventionalisierten syntaktisch-semantischen Potenzials beruht. Beispielsweise kann der Gegenstand der Lüge im Englischen kaum durch einen that-clause ausgedrückt werden (Fischer 1997: 241), während eine entsprechende Konstruktion im Ungarischen normal ist, vgl.

(7) ?He lied that he was poor.

(8) Azt hazudta, hogy szegény. daspron.Acc log-er dasssubj arm

3. Die DG ist eine datennahe Syntaxtheorie einerseits in dem Sinne, dass sie sich nicht auf einige abstrakte Strukturen beschränkt, sondern dass sie einen erheblichen Ausschnitt von Sprache darstellen möchte. Andererseits in dem Sinne, dass sie einen Abstraktheitsgrad anstrebt, der eine methodisch kontrollierte Rückbindung an die Daten ermöglicht. Dependenzgrammatische Beschreibungen können falsifiziert werden.

4. Der wortgrammatische Ausgangspunkt macht die DG für maschinelle Sprachverarbeitung (Parsing, Generation, Translation) in besonderer Weise geeignet.

5. Die relative Einfachheit dependenzgrammatischer Darstellungen macht die DG für didaktische Zwecke geeignet. Eine Reihe von Lernergrammatiken und DaFMaterialien benutzt dependenzgrammatische Darstellungen (vgl. Fobbe 2010).

Aus der wortgrammatischen Perspektive ergibt sich, dass die zentrale Frage in der DG die Begründung von Dependenzen ist, d. h. die Motivierung von Konnexionen und deren Gerichtetheit. Beide werden in der modernen DG - viel stärker als bei Tesnière - auch durch Valenzbeziehungen gerechtfertigt (z. B. Engel 1994). Trotzdem sind beide keinesfalls identisch: Die DG ist eine allgemeine syntaktische Theorie, die nach Welke 1995 auf drei Typen von Dependenzrelationen Rektion, Endozentrik und Subkategorisierung - beruhen kann. Die VT ist eine Teiltheorie, die Kombinationspotenzial und -realisierung relationaler Zeichen (Verben, Adjektive, relationaler Substantive) betrifft. Daher lässt die VT sich nicht nur in andere syntaktische Theorien integrieren, ihre Integration ist Gebot: Keine Grammatik mit einem umfassenden Beschreibungs- und Erklärungsanspruch kommt ohne eine Valenzkomponente aus. Warum dies so ist, wird im folgenden Kapitel ausgeführt, in dem eine moderne VT begründet und begrifflich ausgestaltet wird. 
2. Valenztheorie

2.1 Einordnung

Die menschliche Sprache konstruiert ihre Aussagen nicht ganzheitlich, sondern in Prädikat-Argument-Strukturen: Geschehen werden aufgefasst als aus Dingen und Beziehungen zwischen diesen Dingen bestehend. Nomina referieren auf Entitäten, zwischen denen ein Verb als relationaler Ausdruck eine Verbindung stiftet. Die Denotate von Verben sind abstrakt und nur indirekt greifbar: Man kann z. B. auf sie nur zeigen, indem man auf einen oder mehrere Gegenstände zeigt (vgl. Leiss 2002).

Alle Sprachen besitzen sprachliche Mittel, um Prädikat-Argument-Strukturen zu schaffen (Propositionsbildung). Die VT untersucht diesen Kern menschlicher Sprache sowohl als Universale als auch in seiner einzelsprachlichen Ausprägung.

Die moderne VT geht zwar auf Tesnière zurück, aber der Valenzgedanke ist viel älter. Als unmittelbare Vorläufer werden u. a. K. Bühler (1934) und J. W. Meiner (1781) genannt, aber Vorläuferkonzepte sind schon in der Antike, der arabischen grammatischen Tradition und bei den mittelalterlichen Modisten identifiziert (Seidel 2003, Owens 2003 und Seppänen 2003). Zu erwähnen wäre auch die mehrstellige Prädikatenlogik Freges (1879), die die einstellige, auf der SubjektPrädikats-Opposition beruhende aristotelische Logik ablöste. Sowohl Tesnière als auch seine Nachfolger sehen sich in der Tradition des europäischen Strukturalismus, aber dass die VT zunächst vor allem in Mittel- und Osteuropa weiterentwickelt wurde, ist nicht nur wissenschaftshistorisch, sondern auch in der offensichtlichen Anwendbarkeit auf Kasussprachen motiviert. Mittlerweile gibt es Forschungen zu einer Vielzahl von Sprachen, insbesondere auch zu den romanischen Sprachen und zu Englisch. Die Anzahl der Veröffentlichungen zur Valenz ist auf ca. 3000 Titel zu schätzen.

Die Entwicklung der VT fand nicht isoliert von den in der zweiten Hälfte des 20. Jahrhunderts dominierenden, vor allem angelsächsischen linguistischen Ansätzen statt: Zu erwähnen sind hier die semantische Merkmalanalyse von J. Katz und J. Fodor (1963), das Rollen-, später Szenenkonzept C. Fillmores (1968, 1977), die generelle Hinwendung zu semantisch-pragmatischen Ansätzen in den 70er Jahren (generative Semantik, funktionale Ansätze), kategorialgrammatische Ansätze, die typologische Öffnung der VT im letzten Jahrzehnt und die jüngste Auseinandersetzung mit konstruktionsgrammatischen Ideen (Welke 2009a und 2009b). Verwandte Anliegen sehen Valenztheoretiker in (anderen) wortgrammatischen (Hudson 2006, Starosta 1988) und funktionalen Ansätzen (Halliday 1994, Dik 1978, Givón 1995).

Dieser Offenheit für außerhalb der VT entwickelte Konzepte steht eine geringere Rezeption der VT durch andere Ansätze gegenüber (s. aber Huddleston/ Pullum 2002). Allerdings besitzen heute wohl alle Grammatiken der VT entsprechende Komponenten, meist unter anderem Namen (z. B. Subkategorisierung), 
wobei die Analysetiefe erheblich variiert. Auch haben sich diese Valenzkomponenten zunehmend der VT angenähert, was z. B. in dem X-bar-Schema der generativen Grammatik deutlich wurde (Chomsky 1970, Jackendoff 1977).

Die VT beruht auf der ganz einfachen Idee bzw. Beobachtung, dass Wörter ihre syntaktische und semantische Umgebung bestimmen:

(9) Der Mann wäscht sein Hemd.

Das Verb waschen eröffnet zwei Leerstellen, die eine ganz bestimmte kategoriale Füllung in einer bestimmten Funktion verlangen. Das Verb legt der ersten Stelle die Funktion eines Waschenden (Agens) und der zweiten Stelle die Funktion des Gewaschenen (Patiens) auf: Die Waschen-Szene wird damit vom Waschenden aus gesehen, das Verb waschen eröffnet eine Perspektive auf die Waschen-Szene (Welke 2005).

Die funktionale Forderung des Verbs bestimmt weitgehend die kategoriale Füllung der Leerstellen. In wörtlicher Verwendung können nur Entitäten mit dem Merkmal ,materiell‘ waschen oder gewaschen werden. Und nur eine Entität, die eine Aktivität ausführen kann, kann waschen. Entsprechend ist auch die kategoriale Füllung auf der sprachlichen Ebene beschränkt: Subjekt und Objekt müssen als Nominalgruppe realisiert werden, nicht etwa als Präpositionalgruppe.

Die semantischen Restriktionen sind hier ein direkter Reflex unseres Wissens um Waschvorgänge. Aber Lexeme sind arbiträr in dem Sinne, dass die Realität uns nicht zwingt, einen bestimmten sprachlichen Begriff zu bilden. Ein Bezug auf Waschvorgänge wäre auch durch ein generisches Verb und eine Adverbialgruppe zu erreichen (mit Wasser reinigen). Auch die genaue Eingrenzung dessen, was man mit dem Verb waschen bezeichnet, ist nicht durch die Realität vorgegeben. Man wäscht die Hände, aber nicht die Zähne. Das Säubern des Fußbodens mit Wasser wird eher als den Boden wischen als den Boden waschen angesprochen, während das waschen entsprechende englische Verb geläufiger ist (wash the floor). Berücksichtigt man auch die metaphorische Verwendung, so wird wash enger benutzt als waschen (Geld waschen vs. launder money, vgl. Feilke 1998: 75).

Verben werden nicht nur mit Aktanten, sondern auch mit Angaben kombiniert:

(10) Der Mann wäscht sein Hemd in der Küche.

Die Präpositionalgruppe in der Küche situiert die Waschen-Handlung. Zwar wissen wir, dass alle physischen Vorgänge an einem Ort und zu einer Zeit stattfinden, aber hier handelt es sich um Weltwissen und nicht um eine Forderung des Lexems waschen: Satz (9) oben stellt eine vollständige sprachliche Realisierung des im Verb waschen angelegten Szenarios dar, ohne dass eine örtliche und zeitliche Information schon mitverstanden ist. ${ }^{7}$ Anders in

7 Der Begriff $S_{\text {zenario }}$ bezieht sich auf sprachlich entworfene Sachverhalte, nicht auf die außersprachliche Realität. 
(11) Er legt sein Hemd in die Küche.

(12) *Er legt sein Hemd.

Hier ist die Ortsbestimmung konstitutiv für das legen-Szenario: legen eröffnet eine Leerstelle für eine Ortsbestimmung, ohne deren Füllung kein minimales legenSzenario realisiert werden kann.

Eine Valenzbeschreibung des Kombinationspotenzials des Verbs waschen kann folgendermaßen aussehen:

(13) waschen <AGENS: material (Lebewesen, Institution, Maschine) $\rightarrow$ Subjekt: NP; PATIENS: material $\rightarrow$ Akkusativergänzung $: \mathrm{NP}>$

Die VT betont, dass die verschiedenen syntaktischen Funktionen etwas gemeinsam haben, nämlich Leerstellen eines Verbs zu füllen. Deshalb werden sie einschließlich des Subjekts unter dem Begriff Aktant subsumiert, obwohl sie in anderer Hinsicht ganz verschieden sind.

Man kann vier Grundfragen der V'T identifizieren (Ágel 2000: 115ff.):

1. Was zählt als Valenzträger?

2. Was ist das Ergänzungspotenzial des Valenzträgers?

3. Welche Formen und Typen der Valenzrealisierung gibt es in verschiedenen Sprachen?

4. Wie gestaltet sich die Realisierung von Valenz in Texten?

Wir diskutieren in den Abschnitten 2.2-2.5 die Grundfragen 1 bis 3. Grundfrage 4 wird aus Platzgründen nur marginal berührt, um in den Abschnitten 2.6 bis 2.8 auf die Valenz anderer Wortarten, den Valenzwandel und die Valenzpraxis bzw. angewandte Valenzforschung eingehen zu können.

\subsection{Valenzpotenz: der Valenzträger}

\subsubsection{Identifizierung des Valenzträgers}

Die Frage nach der Valenz (oder nach den Valenzrelationen) von X setzt voraus, dass wir X (den Valenzträger) kennen:

(14) Die Frau hat ihre Bluse gewaschen.

Ist das relationale Sprachzeichen in (14) das Systemelement waschen oder eine Instanz des Wortes waschen im Text (etwa gewaschen oder hat gewaschen)? Gehen wir von der Valenz des Wortparadigmas waschen aus oder von verschiedenen je nach Teilparadigma (z. B. Aktiv, Passiv) oder gar je nach Wortform? Handelt es sich bei einem polysemen Verb wie angehen (,zu funktionieren beginnen', ,etwas in Angriff nehmen', ,angreifen', ,betreffen'; s. unten) um einen Valenzträger oder um mehrere? Sind nur Vollverben Valenzträger oder auch Modal- und Kopulaverben (Hyvärinen 2003: 743ff.)? 
Weiterhin muss bestimmt werden, wie mit idiomatischen Ausdrücken umgegangen wird. Ist der Valenzträger von

(15) Er hat ins (?grüne) Gras/?in den Rasen gebissen. (,sterben?)

der unanalysierte einwertige Valenzträger ins Gras beißen? Oder soll eine von der Valenz von beißen abgeleitete innere Valenz im idiomatischen Ausdruck angenommen werden? Eine Ausgrenzung von Idiomatik aus der VT ist insofern nicht realistisch, als einerseits uneingeschränkt freie Kombinierbarkeit eher die Ausnahme ist, andererseits auch idiomatische Ausdrücke begrenzte Variabilität erlauben (s. Ágel 2004):

(16)(a) Das/?Sie geht (mir) (schon)(sehr/?wenig) an die Nieren.

(b) (?)Das/Sie geht mir (schon) (sehr/?wenig) auf die Leber.

(c) Das/?Sie schlägt (mir) (schon) (sehr/?wenig) auf den Magen.

Weitere Abgrenzungsprobleme ergeben sich bei bestimmten Partikelverben (s. 2.5.2), insbesondere im Englischen auch bei Präpositionsverben: Gehört after (,nach) in look after sbdy (,auf jmdn. aufpassen') zum Valenzträger oder zur Ergänzung? Regiert das Verb look after ein Direktes Objekt oder das Verb look ein Präpositionalobjekt (vgl. Quirk et al. 1985, Emons 1974)?

Diesen Problemen versucht man einerseits mit einer differenzierten Valenzträger-Typologie (s. Ágel 2000: 113ff.), andererseits mit dem Konzept der strukturellen Valenzrealisierung (s. unten) zu begegnen.

\subsubsection{Das Lesartenproblem}

Neben dem externen Identitätsproblem wie der Frage nach dem Valenzträger bei verbalen Idiomen oder Partikelverben existiert auch ein internes Identitätsproblem. Bei den meisten Verben geht nämlich die einfache Rechnung 1 Valenzträger $=1$ Verb nicht auf, da sie mehrere Lesarten besitzen (Variantenproblem s. Ágel 2000: 115ff.):

(17) Das Licht geht an. (,zu funktionieren beginnen?)

(18) Sie geht ihre Aufgabe an. (,in Angriff nehmen)

(19) Sie geht ihren Chef an. (,angreifen')

(20) Das geht uns alle an. (,betreffen')

Zwar sind (17), (18) und (19) inchoativ, aber (20) ist durativ. Ein gemeinsames Bedeutungselement zwischen den Lesarten ist synchron nicht erkennbar. Trotzdem handelt es sich nicht um einen Fall von Homonymie, da es im Sinne des Familienähnlichkeitskonzepts semantisch-metaphorische Verbindungen zwischen den Lesarten gibt: (17) und (18) markieren den Anfang von etwas, (18) und (19) indizieren eine Art Angriff, (18), (19) und (20) sind über den hohen Grad der Affizierung des Patiens bzw. Experiens verbunden. Erwähnenswert ist auch, dass 
die Partikel an nicht durchgehend Akkusativierung bewirkt (*Sie geht das Licht an. ,anmachen $)$. Aus diesen Befunden sind drei Konsequenzen zu ziehen:

1. Der Valenzgrundsatz, dass das Verb Zahl und Art der Ergänzungen bestimmt, ist nur aufrecht zu erhalten, wenn von Verblesarten ausgegangen wird.

2. Geht man vom Lexem angehen aus, so kontextualisiert in der Rezeption der aktuell realisierte Ergänzungsrahmen das Verb näher, er bestimmt, welche Lesart vorliegt. Hat die Akkusativergänzung z. B. das Merkmal ,abstrakt', so liegt Lesart (18) vor, während das Merkmal ,anim‘ auf Lesart (19) oder (20) deutet.

3. Valenz erweist sich als „unordentliches“ (messy, Herbst 2007: 26) und deshalb unvorhersagbares Phänomen: Aus der Bedeutung eines Verblexems bzw. einer Verblesart lässt sich nicht immer bzw. nicht vollständig die Liste der mit dem Lexem bzw. der Lesart kompatiblen Satzmuster ableiten. Vielmehr bleibt ein idiosynkratischer Rest, dem ohne die Beschreibung des Einzellexems und dessen Lesarten nicht beizukommen ist.

Das Lesartenproblem geht aber noch tiefer. Wieviele Lesarten besitzt das Verb angehen? VALBU gibt sieben an, das Großwörterbuch Deutsch als Fremdsprache zehn. Der Unterschied hat zwei Gründe: Erstens führt das Großwörterbuch Deutsch als Fremdsprache Lesarten auf, die von VALBU als Idiome behandelt werden (Etwas geht (gerade) noch an (,erträglich sein), Was das angeht [...] (,betreffen $\left.{ }^{6}\right)$ ). Der zweite Grund wiegt schwerer: Nicht alle Lesarten lassen sich aufeinander abbilden! Z. B. unterscheidet das Großwörterbuch Deutsch als Fremdsprache eine Lesart ,beginnen zu brennen' von einer Lesart in Gang kommen', während VALBU beide zusammenfasst. Keines der beiden Wörterbücher führt eine Lesart ,einen biologischen Prozess beginnen' (Die Pflanze/Bakterienkultur ist angegangen) auf. Ist dies eine weitere Lesart oder gehört die Verwendung zu ,zu funktionieren beginnen' (VALBU) oder zu ,irgendwann beginnen' (VALBU)? Offensichtlich sind Lesarten nicht in demselben Maße objektiv gegeben wie z. B. Tierarten, sondern sie stellen (auch) Konstruktionen des Semantikers, Syntaktikers und Lexikographen dar. Je genauer man hinschaut, desto komplizierter wird das Bild, unterschiedliche Einteilungen sind möglich. Verben bestimmen also nicht nur ihre Umgebung, sondern sie werden auch bestimmt: Die Bedeutung von Verben ist häufig abstrakt bzw. sie haben eine Reihe von durch Familienähnlichkeiten miteinander verbundenen Gebrauchsweisen und bedürfen deshalb der Eingrenzung durch den sprachlichen und nichtsprachlichen Kontext. So erklären sich auch die besonders starke zwischensprachliche Variabilität von Verben und ihre satzfinale Stellung in fast der Hälfte aller Sprachen. Letztere ist nur unter Einbeziehung des Lesartenproblems mit der valenztheoretischen Sicht des Verbs als konstruktionsdeterminierenden Elements vereinbar.

Das Verhältnis von inhärenter Lexembedeutung, kombinatorischer Bedeutung und Lesarten wird im Moment in der VT, auch in Auseinandersetzung mit der Konstruktionsgrammatik, intensiv diskutiert (s. Willems/Coene 2006, Coene 2006, Welke 2009a und 2009b). 
2.3 Valenzpotenz: Ergänzungen und Angaben

\subsubsection{Valenz als einheitliches Phänomen}

In der VT wurde lange angenommen, dass Valenz ein einheitliches Phänomen ist. Deshalb wurde die Abgrenzung von Ergänzungen und Angaben (= E/AAbgrenzung) für die Entwicklung einer VT als grundlegend angesehen (zusammenfassend s. Storrer 2003). Erhebliche Forschungsenergie wurde in die Operationalisierung dieser intuitiv so einleuchtenden Unterscheidung gesteckt, ohne dass ein Konsensus hergestellt werden konnte. Das Problem der Abgrenzung besteht einerseits darin, dass nicht alle Ergänzungen obligatorisch zu realisieren sind. Weglassbarkeit ist also kein hinreichendes Kriterium für Angabestatus:

(21) Sie liest ein Buch.

(22) Sie liest.

Die Leerstelle für die Akkusativergänzung von lesen kann nicht nur syntaktisch, sondern auch semantisch ungesättigt bleiben, da das Patiens des Lesens nicht einmal kontextuell gegeben sein muss, um (22) zu verstehen.

Andererseits können dieselben Wortgruppen sowohl Ergänzungen als auch Angaben sein, es gibt also kein einfaches formales Kriterium für die Abgrenzung. Dieselbe Kette kann je nach Verb oder je nach Interpretation des Szenarios Ergänzung oder Angabe sein:

(23) Sie hat in Kassel gewohnt.

(24) Sie hat sich in Kassel das Bein gebrochen.

(25) Sie plante den ganzen Tag.

Wohnen verlangt normalerweise eine zweite Ergänzung (?? Sie hat gewohnt.), die durch die Präpositionalgruppe in Kassel realisiert wird, während dieselbe Phrase in Relation zu sich das Bein brechen einen Umstand des Szenarios benennt, also Angabe ist.

(25) ist syntaktisch ambig, da den ganz̧en Tag als Ergänzung (Akkusativergänzung: Planung des Tagesverlaufs) oder als Angabe (Zeitangabe: Planung von etwas während des ganzen Tages) interpretiert werden kann.

Lösungsversuche (mit entsprechenden Tests) erfolgten u. a. auf morphosyntaktischer (Rektion, Eisenberg 2006: 33ff.), distributioneller (Subklassenspezifik der Ergänzungen, Engel 1994: 99 und 1988: 183) und semantischer Basis (Konstitution einer Minimalproposition: Zifonun et al. 1997: 1029, Fischer 1999 und 2001: 259ff.). Als wenig befriedigende Auswege boten sich ein skalarer oder gradueller E/A-Übergang an (z. B. Heringer 1984 und 1985, Somers 1987). 


\subsubsection{Multidimensionale Valenzmodelle}

Dieser Forschungssituation gab J. Jacobs (1994) eine überraschende Wende. Er behauptete, dass es sieben (später vier) gültige Präzisierungen des Valenzbegriffs gebe, denen voneinander unabhängige Relationen entsprächen: Valenz sei gar nicht ein einheitliches Phänomen, sondern ein cover term für eben diese Valenz̧bindungsrelationen, die allerdings prototypisch zusammen auftreten. Jacobs' Intervention führte zu multidimensionalen (oder modularen) Valenzmodellen, die heute das vorherrschende Paradigma in der VT darstellen (s. auch Jacobs 2003). Die folgende Darstellung orientiert sich an dem multidimensionalen Valenzmodell der IDS-Grammatik (Zifonun et al. 1997: 1026ff.), ohne die einzelnen Relationen diskutieren zu können:

a) Formrelationen
(i) Fixiertheit:
Erverschlang eine Currywurst. *Erverschlang.
(ii) Rektion:
(iii) Konstanz:
Sie hilft ibm/*ibn.
(iv) Kasustransfer:

Das Verb entscheidet, ob und unter welchen Bedingungen eine Ergänzung weggelassen werden kann (i) und welche Formmerkmale sie trägt: in welchem Kasus sie steht (ii) (hier: das Objekt muss im Dativ stehen), welche Präposition ein Objekt anschließt (iii) und bei Präpositionen, die verschiedene Kasus regieren können, welchen Kasus die von der verbspezifischen Präposition abhängige Nominalgruppe besitzt (iv): Die Präpositionen vor und auf können den Dativ oder Akkusativ regieren. Die Wahl ist in (iv) von dem jeweils regierenden Verb abhängig.

Die Formrelationen können je nach Sprache bzw. Sprachtyp unterschiedlich ausgebildet sein. Z. B. kommt für das Englische als Formrelation die Zuweisung unmarkierter Ergänzungspositionen durch das Verb (als Klasse) hinzu. Entsprechend besitzen englische Angaben größere Positionsvariabilität als englische Ergänzungen, was für die E/A-Abgrenzung nutzbar gemacht werden kann (s. Huddleston/Pullum 2002: 225ff.). Auch für das Deutsche ist eine Formrelation Position relevant, da Lokalergänzungen im Gegensatz zu Lokalangaben, zumindest tendenziell, nach Modalangaben auftreten (Durrell 2010: 149):

(26) Sie ist schnell in die Vorlesung gegangen/ ?in die Vorlesung schnell gegangen. vs.

(27) Sie hat in der Vorlesung schnell geschrieben/ ?schnell in der Vorlesung geschrieben.

b) Bedeutungsrelationen

(v) Sachverhaltsbeteiligung:

Sie kommen zu Hause an.

(vi) Perspektivierung: Das Auto kostet 4000 Euro. Vgl. Sie kauft das Auto für 4000 Euro.

(vii) Synsemantische Kodierung: Er wartet auf den Bus. Vgl. Er springt auf den Bus. 
Um einen Sachverhalt des Ankommens zu entwerfen, ist Bezug auf eine Entität, die ankommt, und auf einen Ort, an dem sie ankommt, erforderlich (v). Das Verb kosten perspektiviert den Preis stärker als das Verb kaufen (vi). Die semantische Beziehung zwischen dem Verb warten und dem Objekt des Wartens wird nicht durch die räumliche Bedeutung von auf hergestellt, sondern ist von dem Verb abhängig (vii). Im Gegensatz dazu wird die Beziehung zwischen dem Verb springen und der Akkusativphrase (den Bus) durch die Bedeutung von auf hergestellt.

Je mehr Relationen eine Wortgruppe auf sich vereint, in umso höherem Grade handelt es sich um eine Ergänzung. Prototypische Ergänzungen und Angaben zeigen Konvergenz der Form- und Bedeutungsrelationen. Zwischen den Relationen gibt es implikative Beziehungen, z. B. folgt aus Sachverhaltsbeteiligung starke oder schwache Perspektivierung.

Kritisch wurde zu multidimensionalen Valenzmodellen angemerkt, dass die binäre Teilung in Ergänzungen und Angaben ein unhintergehbares, psychisch reales Grundphänomen ist (Fischer 2001, Welke 2009a) und dass die Relationen nicht gleichrangig sind. Eine geeignet definierte Relation ,Sachverhaltsbeteiligung begründet Valenz als universelles Phänomen und entscheidet in hohem Maße über den Ergänzungsstatus (vgl. Fischer 1999, 2001). Die Formrelationen betreffen dagegen die einzelsprachliche Realisierung von Valenz, von der auch die beiden anderen Inhaltsrelationen abhängen: Sie müssen je nach Sprache auch z. T. anders gefasst werden. Für das Englische muss z. B. eine Formrelation Position hinzugefügt werden (s. 1.3), während die Relation Kasustransfer synchron keine Rolle mehr spielt.

\subsubsection{Ergänzungen und Argumente}

Obwohl Valenz in Prädikat-Argument-Strukturen, also semantisch-pragmatisch, begründet ist, ist sie nicht mit diesen gleichzusetzen. Nicht alle Argumente werden als Ergänzungen realisiert, und nicht alle Ergänzungen sind Argumente. Z. B. besitzen Adjektive eine Argumentstelle, die nicht als Ergänzung in der Adjektivgruppe, sondern vom Bezugsnomen gesättigt wird (eine stolze Frau, sie ist auf ibre Kinder stoly). Semantische Valenzstellen können auch als Teil der Lexembedeutung realisiert werden oder sonstwie blockiert sein: Bei Verben wie hämmern, nageln ist die Argumentstelle des Instruments in das Verb inkorporiert, eine Reihe deutscher Partikelverben erlaubt keine Nennung des Patiens (z. B. zuschlagen: *Er schlägt ibn zu.), das aber mitverstanden wird.

Hier zeigt sich auch, dass die VT nicht auf den Prädikatenkalkül reduzierbar ist, obwohl beide in den in sprachlichen Äußerungen enthaltenen Propositionen ihren Ausgang nehmen. 


\subsection{Valenzänderung}

\subsubsection{Grundvalenz und Valenzänderung}

Für ein Verb wie waschen lässt sich überzeugend darstellen, dass die Lexembedeutung die Valenz bestimmt (s. 2.1). Die zweiwertige Realisierung der Valenz von waschen kann als Grundvalenz angesprochen werden (vgl. Welke 1988). Auch Valenzänderungen (Valenzreduktionen und -erhöhungen) sind zumindest erwartbar: die (idiomatisch geprägte) indefinite Verwendung (Er wäscht , macht die Wäsche'), ${ }^{6}$ die mediale Verwendung mit Medialmarker sich (Sie wäscht sich), ${ }^{9}$ die sekundäre Subjektivierung des Patiens, ebenfalls mit Medialmarker sich (Diese Hemden waschen sich gut), die resultative Verwendung (Er wäscht seine Kleider zu Fetzen), die medialresultative Verwendung (Sie wäscht sich noch z $u$ Tode), ${ }^{10}$ die instrumentale Verwendung (Sie wäscbt ibren Wagen mit dem Gartenschlauch), die benefaktische Verwendung (Sie wäscht ibm den Wagen), die benefaktiv-resultative Verwendung (Sie wäscht ihm den Wagen sauber) und die medial-benefaktiv-resultative Verwendung (Sie wäscht sich ibm noch $z u$ Tode). ${ }^{11}$ Das Valenzpotenzial und die Valenzrealisierungen von waschen lassen sich aus der inhärenten Lexembedeutung unter Zuhilfenahme von Weltwissen, lexikalischen und syntaktischen Regeln zur Valenzänderung (vgl. Alternantenproblem bei Ágel 2000: 116-128; s. auch 145ff.) und der Kasushierarchie ableiten.

\subsubsection{Szenarioverändernde Valenzänderungen}

Valenzänderungen wie (a) Sie wäscht ibm den Wagen (Erhöhung), (b) Sie wäscht oder (c) Sie wäscht sich (beides Reduktion) sind sog. szenarioerhaltende Valenzänderungen (Fischer 2003). In (a) wird ein Argument hinzugefügt, in (b) ist ein Argument idiomatisch mitverstanden und seine Realisierung deshalb unüblich (? Sie wäscht Wäsche), in (c) fallen beide Argumente zusammen, d. h. das Agens ist zugleich das Patiens der Handlung. Trotz Valenzänderung handelt es sich in den drei Fällen um ein Waschen-Szenario. Im Gegensatz dazu verändert die Valenzerhöhung in (29) das Szenario in (28):

(28) Der Hund ist weggelaufen.

(29) Ihm ist der Hund weggelaufen.

Während (28) ein Weglaufen-Szenario konstruiert, führt die Erweiterung um eine Dativergänzung zu einem sekundären Verantwortlichkeitsszenario in Bezug auf das

8 Erwartbar ist, dass es eine indefinite Verwendung gibt, nicht, welche idiomatische Bedeutung sie hat. Um über letztere eine Vermutung anzustellen, genügt nicht die Kenntnis der inhärenten Lexembedeutung, sondern Kollokationen und kulturelle Praktiken müssen herangezogen werden.

9 Ohne Kontrastintonation.

10 Diese kann indefinit (,Sie wäscht zuviel Wäsche) oder medial (,Sie wäscht sich zuviel'), also ,doppelt medial', interpretiert werden!

11 Statt einer benefaktivischen wäre auch eine Interpretation als ethischer Dativ denkbar. 
primäre Weglaufen-Szenario. Der Valenzträger weglaufen besitzt keine Leerstelle für eine Dativergänzung: Das Verantwortlichkeitsszenario wird dem Verb oktroyiert.

Das Konzept der szenarioverändernden Valenzänderung (Fischer 2003: 28ff.) entschärft verschiedene Probleme der VT: das Lesartenproblem, das Problem der Verbformenvalenz, das Problem der Abgrenzung von Ergänzungen und Angaben, das Problem der textuell-situativen Valenzrealisierung. Es ist eine Theoriekomponente, die die Integration einer nicht unbedeutenden Gruppe von konstruktionistischen Phänomenen in eine projektionistische Theorie erlaubt (zum Problem s. Abschnitt 4).

\subsection{Strukturelle Valenzrealisierung}

\subsubsection{Formale Realisierung der Valenz}

Tesnière beschränkte die Valenz auf die Zahl der Aktanten. Nach der modernen VT bestimmen Verben Zahl und Art ihrer Leerstellen (z. B. Helbig 1992). Am greifbarsten äußert sich Valenz in der Kennzeichnung der Aktanten durch Kasus:

(30) Der Nom Hund folgt dem Dat Mann.

Im Deutschen muss der Folgende im Nominativ, der, dem gefolgt wird, im Dativ realisiert werden, wobei die Kasuskennzeichnung vor allem am Determinativ erfolgt. Permutation der unmarkierten Reihenfolge Subjekt - Dativergänzung ändert zwar die Informationsstruktur, aber nicht die Aussage:

(30)(a) Dem Mann folgt der Hund.

In Nicht-Kasussprachen können nominale Ergänzungen positional gekennzeichnet sein. Z. B. ist im Englischen parallel zum Kasusabbau die unmarkierte Reihenfolge grammatikalisiert worden:

(31) The dog follows the man.

,Der Hund folgt dem Mann

(32) The man follows the dog.

,Der Mann folgt dem Hund

Der Folgende wird präverbal, der Gefolgte postverbal realisiert. Eine Vertauschung der Positionen ändert im Gegensatz zum Deutschen die Aussage.

Eine weitere formale Kennzeichnung erlauben verbspezifische präpositionale Anschlüsse, die oft als Kasusäquivalent angesehen werden:

(33) Sie besteht auf/*unter $/ *_{\text {in }} / *$ für Service.

Zwei wichtige Fragen ergeben sich in Bezug auf die formale Kennzeichnung von Ergänzungen:

1. Wie eindeutig ist sie?

2. Dient sie nur der Unterscheidung der Ergänzungen, oder transportiert sie auch semantische Information? 
Sowohl Position als auch Morphologie kennzeichnen oft nicht eindeutig: Zwar sind Positionen an sich eindeutig definiert (vor X, nach X), sie sind aber nicht auf die Kennzeichnung einer Funktion festgelegt. Deshalb muss bei der Definition der englischen Ergänzungen von unmarkierten oder kanonischen Positionen ausgegangen werden (s. Huddleston/Pullum 2002: 225f.). Morphologische Kennzeichnungssysteme tendieren zu Formensynkretismus (oft Subjekt-Direktes Objekt; vgl. Plank 1984). Andererseits ist die Zahl der Differenzierungen in Kasussystemen potentiell weniger begrenzt als die Zahl von Positionen.

Es wäre unökonomisch, wenn formale Kennzeichnung nur der Unterscheidung der Ergänzungen diente, aber die Abbildung der formalen Kennzeichnungen auf semantische Rollen ganz idiosynkratisch erfolgte, z. B. ein Agens unmotiviert durch verschiedene Positionen, Kasus oder Präpositionen gekennzeichnet sein könnte. Die Abbildung folgt Regularitäten, die auf konkurrierenden Prinzipien gründen und für die Präsenz anderer Ergänzungen sensibel sind (vgl. die Kasushierarchie in Eisenberg 2006: 68ff. und die Salienzordnung der Beteiligtenrollen in Zifonun et al. 1997: 1300ff., insbes. 1326).

\subsubsection{Mikro- und Makrovalenzrealisierung}

Dass sich eine typologisch adäquate VT nicht mit der Unterscheidung zwischen Valenz als Potenz und Valenz als textuell-situativer Realisierung dieser Potenz (Tesnières valence libre) begnügen kann, hat bereits Tesnière klar gesehen (s. Anm. 2). Die Realisierung der Valenzpotenz hängt nämlich auch von generellen dependenzstrukturellen Eigenschaften von Sprachen, Varietäten bzw. von der zu realisierenden grammatischen Kategorie ab. Beispielsweise werden in den romanischen Sprachen mit Ausnahme des Französischen pronominale Subjekte unmarkiert nicht realisiert:

(34) ital. Cerco una casa.

suche-1.Pers.Sg.Präs. eine Wohnung

,Ich suche eine Wohnung'

Ist das italienische Subjekt partiell fakultativ? Die Frage ist auf der falschen Ebene gestellt. Eine Realisierung des pronominalen Subjekts im Italienischen ist nämlich nicht mit der Realisierung eines pronominalen Subjekts etwa im Deutschen oder Englischen gleichzusetzen. Vielmehr ist sie emphatisch:

(35) ital. Io cerco una casa.

„ICH suche eine Wohnung

Die Nicht-Realisierung pronominaler Subjekte in romanischen Sprachen ist keine Frage von Register, Text, Kontext und Situation, sondern des Typus der strukturellen Valenzrealisierung. (34) ist nicht subjektlos, sondern die Verbform cerco 
fungiert als Valenzträger und Erstaktant in einem. Die Aktantenrealisierung in der Verbform wird seit Pasierbsky 1981 als „Mikrovalenz“ angesprochen.

Strukturell gibt es nach dem Standardmodell zwei Realisierungsmöglichkeiten für Ergänzungen: 1. als in den Valenzträger inkorporiertes Flexiv (Mikrovalenz) 2. als separate Wortgruppe (Makrovalenz). Mikrovalenz ist nicht auf das Subjekt beschränkt (s. László 1988):

(36) ung. Eszi.

$$
\text { ,er/ sie/es-isst-es }
$$

Eszi realisiert sowohl Subjekt als auch Direktes Objekt mikro-, aber nicht makrovalenziell. Die naheliegende Erklärung, dass pronominale Mikrovalenz die unmarkierte makrovalenzielle Nicht-Realisierung bewirkt, greift zu kurz. Einerseits verlangen Sprachen mit Mikrorealisierung des Subjekts (Deutsch, Isländisch) die Makrorealisierung pronominaler Subjekte, andererseits erlauben Sprachen ohne Mikrorealisierung von Subjekt und Objekt (Chinesisch, Koreanisch, Japanisch) pronominale Nicht-Realisierung dieser Ergänzungen.

Die Beispiele zeigen, dass die Dependenzrelationen zwischen Mikro- und Makroebene je nach Aktant und je nach Sprache unterschiedlich sein und daher für eine typologische Einteilung genutzt werden können.

Das Konzept der Mikrovalenz ist über Subjekt und Objekte hinaus auf adverbiale Ergänzungen angewandt worden (Ágel 2000: 138ff.):

(37) Sie hat das Rad an den Zaun gelehnt.

(38) Sie hat das Rad angelehnt.

(39) Sie hat das Rad an den Zaun angelehnt.

(40) Sie hat das Rad daran / an ibn angelehnt.

Der Valenzträger in (37), lehnen, verlangt eine adverbiale Ergänzung. Für (38) wird traditionell anlehnen als Valenzträger angenommen, der fakultativ mit einer adverbialen Ergänzung kombiniert werden kann. Nach Ágel (2000: 138ff.) ist der Valenzträger aber auch in (38) lehnen: Dessen adverbiale Ergänzung muss nicht makrorealisiert werden, sie ist in Form der Partikel an den Valenzträger angeschlossen („head movement“, Nichols 1986). Partikel und Valenzträger bilden zusammen die Verbform, d. h. die adverbiale Ergänzung ist in das Verb inkorporiert. Der Fall ist aber nicht ganz parallel zur Mikrorealisierung in (34) und (36), da die pronominale Makrorealisierung in (40) nicht unbedingt emphatisch ist (vgl. Fischer 2003: 49f.). In stärker kopfmarkierenden, z. B. den nordamerikanischen, Sprachen findet Mikrorealisierung einer Reihe von (auch adverbialen) Ergänzungen und Angaben statt (vgl. Nichols 1986).

Das Konzept der strukturellen Valenzrealisierung ist mit dem Pro-dropParameter der generativen Grammatik verwandt, ist aber älter als dieser. Im Kern geht das Konzept auf Tesnière zurück (s. Ágel 1993). 


\subsection{Valenz anderer Wortarten}

Im Gegensatz zu der auf Verb-Substantiv-Dependenzen operierenden Exklusivität der Valenzrelation bei Tesnière, der nichtverbale Wörter als Valenzträger ausschließt, rechnet man in der modernen Valenztheorie mindestens auch mit adjektivischen und substantivischen Valenzträgern (Groß 2003, Teubert 2003, Hölzner 2007), evtl. auch mit synsemantischen Valenzträgern wie Präpositionen und Subjunktoren (Colliander 1999).

In der Theorie der Substantivvalenz existieren zurzeit drei konkurrierende Ansätze (System sui generis, Nominalisierungsansatz, Stützverbgefüge-Ansatz), von denen nur der Nominalisierungsansatz davon ausgeht, dass es keine genuine Substantivvalenz gebe, sondern dass die Valenzstruktur des Substantivs vom Verb geerbt sei. Dieser mit der X-bar-Theorie vergleichbare Ansatz gilt in der VT mittlerweile als widerlegt (Teubert 2003).

Das multidimensionale Valenzmodell Jacobs'scher Prägung ist inzwischen auch für die Substantivvalenz entwickelt worden (Hölzner 2007).

\subsection{Valenzwandel}

Wie in 1.4 erwähnt, legt die VT nicht nur auf die typologische, sondern auch auf die sprachbistorische Adäquatheit der Theoriebildung, d. h. auf das Prinzip der Viabilität (Ágel 2001), großen Wert. Nach diesem Prinzip muss jede linguistische Beschreibung oder Erklärung eines Phänomens mit der linguistischen Beschreibung oder Erklärung der Geschichte des Phänomens konform sein. Der Theorieapparat muss also idealerweise so beschaffen sein, dass er (a) mit beliebigen Sprachstufen umgehen kann und dass sich (b) die synchronen Beschreibungen der Sprachstufen derselben Sprache in ein diachrones Modell integrieren lassen, dessen natürlicher aktueller ,Endpunkt ${ }^{`}$ der jeweils jüngste Sprachzustand ist.

Seit der zweiten Hälfte der 70er Jahre wurden nicht unerhebliche Forschungsenergien in die valenzielle Untersuchung historischer Sprachstufen (vor allem des Deutschen, aber auch anderer Sprachen) gesteckt (vgl. Kap. XII von Handbuch 2006). Weniger befriedigend ist die Situation bei der diachronen Modellierung des Valenzwandels (zusammenfassend Heringer 2006 und Korhonen 2006). Hier tauchen gravierende empirische, methodische und theoretische Probleme auf, sobald man etwa versucht die für moderne Sprachstufen entwickelten Konzepte der E/A-Abrenzung oder der Identifizierung des Valenzträgers auf ältere Sprachstufen zu übertragen (Habermann 2007). Habermanns Diagnose einer im Vergleich zum Nhd. viel stärkeren Interaktion von Verbvalenz und -bedeutung mit verbunabhängigen Konstruktionen und textuell-pragmatischen Faktoren im Ahd. und Mhd. und die im Laufe der deutschen Sprachgeschichte nachweislich zunehmende Wohlbestimmtheit von Wortbedeutungen (Reichmann 1988: 171) 
legen den vorsichtigen Schluss nahe, dass ältere Sprachstufen stärker konstruktionistisch und weniger projektionistisch zu beschreiben sein dürften als moderne Schriftsprachen. Eine besondere Herausforderung für eine viable Theoriebildung besteht also darin, dass mit einer stabilen Architektur konstruktionistischer und projektionistischer Theoriekomponenten ein historisch instabiles Gefüge von konstruktionistisch und projektionistisch beschreibbaren grammatischen Phänomenanteilen modelliert werden muss.

2.8 Valenzpraxis und angewandte Valenzforschung

\subsubsection{Einordnung}

Die Theoriebildung in der VT wurde wesentlich durch ein (a) einzelsprachliches und (b) kontrastives Beschreibungsinteresse vorangetrieben. Dabei gilt für die Valenzforschung die gängige (und oft missbrauchte) Gegenüberstellung von Theoretischer und Angewandter Linguistik nur sehr eingeschränkt. Denn die Gegenstände der Valenztheorie werden vielfach in der Valenzpraxis, etwa bei der lexikografischen oder kontrastiv-grammatischen Arbeit, erst geschaffen.

In der wissenschaftlichen Diskussion versteht man unter Anwendung ,die Umdeutung eines Mittels bezüglich seines Zwecks“, d. h., ein „für einen bestimmten Zweck A bewährtes Mittel X wird für einen neuen Zweck B zum Mittel Y.“ (Janich 2009: 157) Anwendung in der Valenzforschung hieße demnach Folgendes:

die für die Beschreibung einer wohldefinierten Teilmenge syntaktischer Regularitäten einer Einzelsprache (= A) bewährte Valenztheorie (= X) wird für die Zwecke der lexikografischen oder kontrastiven Beschreibung $(=\mathrm{B})$ zum Beschreibungsinstrument $(=\mathrm{Y})$

Dieser Begriff der Anwendung greift zwar bei einer Reihe von lexikografischen oder kontrastiv-grammatischen Valenzarbeiten, aber bei weitem nicht bei allen. In zahlreichen Fällen tritt an die Stelle des Modells „X als Y anwenden“ das Modell:

durch Y (qua Schnittmenge der Zwecke A und B) zum X beitragen

Diese Art von Beschäftigung mit Valenz nennen wir Valenzpraxis. Beispiele für diese die Valenztheoriebildung ändernde Valenzpraxis sind Legion. Hier seien nur drei genannt:

1. die Auflösung der E/A-Abgrenzung und ihre anschließende Rekonstitution unter neuen Vorzeichen (z. B. Storrer 1992, Jacobs 1994, 2003, Fischer 2001). Die Problematik der Abgrenzung, wenigstens aber ihre theoretische Relevanz, wurde in konkurrierenden Ansätzen, die sich auf eng umgrenzte Ausschnitte von Sprache stützten, also, nicht-angewandt' arbeiteten, z. T. gar nicht erkannt. 
2. Die Problemkomplexe von Variation, Alternation und Valenzänderung (z. B. Ágel 2000, Fischer 2003, Welke 2005) sowie der Bezug verschiedener Lesarten und Gebrauchsweisen aufeinander, die zu einer Reihe neuer Konzepte führen, u. a. dem Konzept der Grundvalenz (Welke 1988) und des Satzmusterparadigmas (Coene 2006). Diese Problemkomplexe ergeben sich unmittelbar aus der lexikografischen Arbeit.

3. Die Entwicklung einer typologisch adäquaten VT, die die Fixierung auf einzelne syntaktische Mittel (traditionelle VT: Morphologie; Generative Grammatik: Topologie) löst, die semantisch-pragmatische Valenzbegründung von der einzelsprachlichen Beschreibung trennt und eine Integration von Kopfmarkierungen (Mikrovalenz) erreicht (z. B. Tesnière 1976, Ágel 1993, 2000, Fischer 2003, Welke 2005).

\subsubsection{Valenzlexikografie und kontrastive Valenz}

Im Rahmen dieses Artikels ist es nicht möglich, valenzlexikografische und kontrastive Arbeiten vorzustellen oder auch nur ihre Innovationen und Ergebnisse anzusprechen. Als repräsentative Werke seien hier genannt: Wörterbuch zur Valenz. und Distribution (Helbig/Schenkel 1969, 81991) und VALBU - Valenzlexikon dentscher Verben (Schumacher/Kubczak/Schmidt/de Ruiter 2004). Obwohl Deutsch die bei weitem valenzlexikografisch erschlossenste Sprache ist, gibt es auch Valenzlexika zu anderen Sprachen, z. B. A Valency Dictionary of English: A Corpus-based Analysis of the Complementation Patterns of English Verbs, Nouns and Adjectives (Herbst/Heath/Roe/Götz 2004).

Eine Reihe kontrastiver Valenzlexika und Grammatiken wurde erarbeitet. Repräsentativ seien genannt: Valenఇlexikon deutsch-italienisch (Bianco 1996), Kontrastive Grammatik deutsch-serbokroatisch (Engel/Mrazović 1986), Kontrastive Grammatik deutsch-rumänisch (Engel/Isbasescu/Stanescu/Nicolae 1993), Deutsch-polnische kontrastive Grammatik (Engel 1999). Drei Sprachen konstrastiert das OnlineWörterbuch The Contragram Dutch-French-English Contrastive Verb Valency Dictionary (Colleman/Defrancq/Devos/Noël 2004). Zur englisch-deutschen kontrastiven Verbvalenz, auch aus typologischer Perspektive s. Hawkins (1986), Durrell (2002), Fischer $(1997,2007 \mathrm{a}, \mathrm{b})$.

\section{Dependenzgrammatik}

3.1 Einordnung

Der Dependenzbegriff hat eine lange Tradition: Im Westen existiert er implizit in antiken Analysen, explizit als determinatio (Boethius) seit dem 6. Jh., als dependentia seit dem 13. Jh. (Percival 1990). Die historischen Dependenzbegriffe unterschei- 
den sich von denen der strukturellen Linguistik in signifikanter Weise. Wir behandeln nur letztere. In 3.2 werden Merkmale einer reinen DG vorgestellt. In 3.3 wird auf die gängigen Präzisierungen des Dependenzbegriffs eingegangen und werden auch Probleme mit dem Kopfbegriff thematisiert. Abgerundet wird das Kapitel und der Beitrag mit einer Diskussion um die zwei grundlegenden syntaktischen Ordnungsprinzipien ,Dependenz ${ }^{6}$ und ,Konstituenz $z^{6}$. Auf die Vorstellung von einflussreichen Dependenzmodellen (wie z. B. Engel 31994[1977], Eroms 1985, 2000, Mel’čuk 1988) muss aus Platzgründen verzichtet werden.

\subsection{Grundsätze der Dependenz}

Neben Konstituenz stellt Dependenz das zweite syntaktische Darstellungsprinzip dar. Ein drittes ist bisher nicht entwickelt worden (Mel’čuk 1988: 13). In der Praxis greifen Grammatiken auf beide Prinzipien zurück, wobei je nach grundsätzlicher Ausrichtung der Syntax entweder Konstituenz oder Dependenz für das grundlegendere Prinzip gehalten wird. Wir stellen nun Grundsätze einer reinen DG vor, die die Merkmale einer Dependenzdarstellung eines Satzes festlegen:

1. Die Elemente sind Wörter (oder Morpheme) bzw. Wortformen.

2. Genau ein Element ist unabhängig. (Spitze des Dependenzbaums)

3. Alle anderen Elemente sind von einem Element abhängig.

4. Kein Element ist von sich selbst abhängig. (keine Reflexivität)

5. Kein Element ist von mehr als einem Element abhängig. (Verzweigung nur nach unten)

6. Ist ein Element $\mathrm{A}$ von einem Element B, und Element B von Element $\mathrm{C}$ abhängig, so ist $\mathrm{A}$ indirekt von $\mathrm{C}$ abhängig. (Transitivität)

Als Ergebnis der Grundsätze ergibt sich ein aktuelles Stemma wie etwa

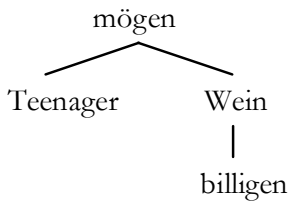

In einem Dependenzbaum kommen nur terminale Elemente vor, keine Knoten bzw. Phrasen, und jene deshalb jeweils nur einmal: Der ganze Baum entspricht dem Satz. Alle Elemente sind miteinander verbunden, die Abhängigkeit vererbt sich entlang der Äste nach unten. Z. B. ist billig nicht nur direkt von Wein abhängig, sondern indirekt auch von mögen (aber nicht etwa von Teenager, da das Wort zwar höher, aber nicht auf demselben Ast angesiedelt ist). Die vorgestellten Prinzipien erlegen reinen Dependenzdarstellungen erhebliche Restriktionen auf. Sie können nur für die Darstellung von Teilausschnitten natürlicher Sprachen durch- 
gehalten werden. Z. B. wird Grundsatz 5 bei der Darstellung der Junktion verletzt: Schon Tesnière (1976: 340) sieht hier multiple Regentien vor.

Dependenzgrammatiken sind Wortgrammatiken: Die für die Dependenzstruktur verantwortlichen Eigenschaften sind im Lexikon aufgeführt, z. B. die Bedeutung, Wortklassenzugehörigkeit, Valenz und andere distributionelle Beschränkungen. Die im Lexikon aufgeführten Informationen können selektiv dazu benutzt werden, die Nuclei abstrakter zu besetzen (s. z. B. Engel 1994, Heringer 1996):

(41)(b)

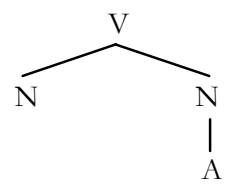

Das virtuelle Stemma (41b) repräsentiert auch mit (41a) strukturell verwandte Sätze wie Kinder essen grünes Gemüse. Beide Darstellungen können kombiniert und durch weitere Lexikoninformationen angereichert werden: ${ }^{12}$

$(41)(\mathrm{c})$

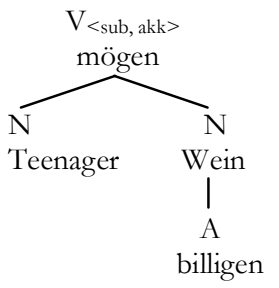

Schließlich können die zwischen den Wortformen bestehenden Dependenzrelationen an den Dependenzkanten näher spezifiziert werden (vgl. Mel’čuk 1988: 16ff.; 2003: 189ff.):

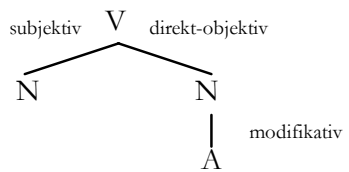

Dependenzbäume erlauben zwar keine Phrasen als Elemente, diese sind aber ableitbar: Ein Element und alle von ihm direkt oder indirekt abhängigen Elemente bilden seine Phrase (Knoten). So bildet Wein und das von ihm abhängige Adjektiv billigen die Nominalgruppe billigen Wein.

Es ist nicht Teil des Grundsatzsystems, dass das oberste Element das Verb ist, aber in vielen klassischen Dependenzdarstellungen ist dies der Fall. Der gesamte Baum ist dann ein Verbalknoten, der identisch mit dem Satz ist, der demnach

$12 \mathrm{sub}=$ Subjekt, akk $=$ Akkusativergänzung 
endozentrisch ist. Auch wenn das Subjekt als Makroaktant als von dem verbflexivischen Mikroaktanten abhängig aufgefasst wird (Eroms 1985, 2000: 86), bleibt die Endozentrik erhalten.

Die Diagramme (41a-d) zeigen nicht die Reihenfolge im Satz. Dies wurde in den auf Tesnière basierenden Darstellungen als Vorteil gesehen, da Dependenzdarstellungen nicht auf die lineare, sondern auf die strukturale Ordnung des Satzes zielten. Allerdings können Dependenzdiagramme projektiv angeordnet werden (vgl. Heringer 1996, Eroms 2000):

(41)(e)

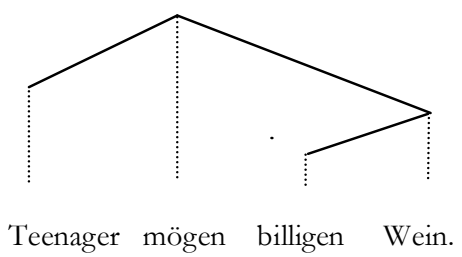

\subsection{Begründung von Dependenz}

Unter ,Begründung von Dependenz ' lässt sich zweierlei verstehen. Einerseits die grundlegende Frage nach der empirischen Fundierung und der psychologischen Realität von Dependenzrelationen. Diese Art von Begründung konnte zugunsten der DG geleistet werden (Leiss 2003). Andererseits geht es um die theorieinterne Begründung von ,oben ‘ und ,unten ‘ in einer Dependenzstruktur. Entscheidend dabei ist, wie bei zwei durch (strukturell oder lexikalisch geregeltes) Miteinandervorkommen als zusammengehörig ausgewiesenen Elementen bestimmt wird, welches das Regens bzw. der Kopf ist. Wir stellen nun die Relationstypen vor, mit denen versucht wurde, den Begriff der Dependenz (= D) zu präzisieren (s. Welke 1995):

1. D1 Endozentrik: Regens ist Voraussetzung für das Vorkommen des Dependens.

2. D2 Subkategorisierung: Regens impliziert ein Set von Dependentien, deren syntaktische und semantische Funktionen es festlegt.

3. D3 Rektion: Regens legt bestimmte formale Eigenschaften (Kasus, Status, kanonische Position, Prosodie) fest.

D1 greift insbesondere für fakultative Elemente:

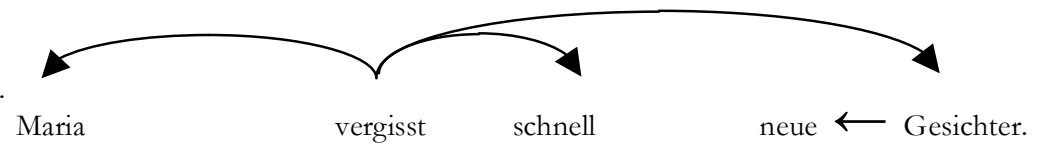


Das Vorkommen des Nomens Gesichter ist Voraussetzung für das Vorkommen des Adjektivs neue. Das Vorkommen des Verbs vergessen ist Voraussetzung für das Vorkommen des Adverbs schnell. In beiden Fällen ist das Dependens im Gegensatz zum Regens weglassbar.

Probleme hat D1, wo beide Elemente obligatorisch sind und sich also gegenseitig bedingen, z. B. Artikel und Nomen (der Wagen) oder Präposition und Pronomen (mit ihr). Dies gilt auch für das Vorkommen von vergisst, das zwar Voraussetzung für das Vorkommen der Nomina Maria und Gesichter ist, aber der umgekehrte Fall gilt auch: Da das Deutsche strukturell eine Makrorealisierung der Ergänzungen verlangt, ist das Vorkommen der beiden Nomina Voraussetzung für das Vorkommen des Verbs vergisst. Zwischen dem Verb und seinem Ergänzungsrahmen müsste nach D1 also Interdependenz angesetzt werden, was im Rahmen der oben angeführten Grundsätze nicht darstellbar ist.

Hier greift D2: Das Verb impliziert das Vorkommen der beiden Nomina, gehört also zu der Subkategorie der zweiwertigen Verben. Dies bedeutet aber, dass D2 Valenzbeziehungen benutzt, um die Abhängigkeitsrichtung zu rechtfertigen: Valenzträger sind zugleich auch Regentien im Abhängigkeitsbaum. Aber nicht alle in einem Satz vorkommenden Dependentien sind impliziert (Angaben, Partikeln). Daher bliebe ein allein auf D2 gegründeter Dependenzbaum unvollständig.

D3 stützt z. T. die durch 1. und 2. ausgewiesenen Dependenzen, kann ihnen aber auch widersprechen $\left(\leftarrow_{\text {form }}=\right.$ formale Rektion $):{ }^{13}$

(43) frz. $\quad$ belles $\leftarrow$ form filles

(44) lat. $\quad$ VicinoDat $\leftarrow$ form favet.

dem Nachbarn bevorzugt-er/sie.

,Er/sie bevorzugt den Nachbarn.

(45) persisch kûhe $\leftarrow$ form boländ (Nichols 1986: 58)

Berg hoch

,hoher Berg'

(46) Das Auto $\rightarrow$ form fährt gut.

Belles ist nach 1. von filles abhängig, aber auch nach 3., da das Genus von filles (Femininum) bewirkt, dass das Adjektiv belles in der femininen Form erscheint (nicht beaux). Vicino ist nach 2. von favet abhängig, aber auch nach 3., da favere den Kasus von vicino (Dativ) bestimmt. In (45) ist das Adjektiv boländ nach 1. von kûhe abhängig, aber die Existenz eines Attributs bewirkt, dass das Nomen kûh das Suffix -e erhält, weshalb boländ formal kûhe regiert. Nach 2. ist das Nomen Auto von fährt abhängig, aber der Singular Auto bewirkt, dass fährt als Singular markiert ist. Generell laufen Kopfmarkierungen wie in (45) und (46) den nach 1. und 2. ausgewiesenen Dependenzmarkierungen entgegen. Werden Dependenzbeziehungen rein auf morphologischer Rektion gegründet, ändert sich die Richtung der Bezie-

13 Wir beschränken uns hier (a) auf den (üblicheren) formalen Rektionsbegriff und (b) auf die Diskussion morphologisch markierter Rektion. Im Prinzip schließt formale Rektion auch die Determination von Topologie und Prosodie ein. 
hung entsprechend dem morphologischen Sprachtyp (kopf- vs. dependensmarkierend). Auch führt ein rein morphologischer Dependenzbegriff nicht zu einem vollständigen Baum, in dem alle Elemente miteinander verbunden sind (vgl. Mel'čuk 1988: 109):

(47) $\mathrm{Il} \rightarrow$ form $\mathrm{a} \rightarrow$ form dormi dans sa $\leftarrow$ form chambre.

,Er hat in seinem/ihrem Zimmer geschlafen`

Schließlich können die Rektionsrelationen je nach beteiligten grammatischen Kategorien komplex und gegenläufig sein: In einer Substantivgruppe wie den $n_{\text {Acc }}$ billigen $_{\text {Acc }}$ Wein, bei der man gewöhnlich annimmt, dass das Determinans (den) der Kopf und das Substantiv (Wein) der Kern ist, kann man drei verschiedene Rektionsrelationen $(=\mathrm{R})$ ausmachen:

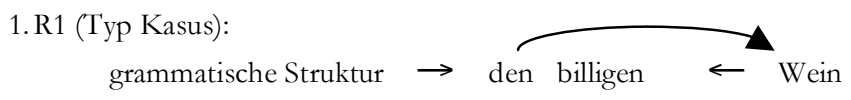

Regens ist der Kopf, direktes Rektum der Kern, indirektes Rektum das Adjektiv. Der Kasus wird extern durch Rektion bestimmt: Der Kopf ist selber ein Rektum, dessen Regens in der jeweiligen grammatischen Struktur zu suchen ist. Primär ist der Kopf morphologisch markiert (den vs. der, des, dem).

2. R2 (Typ Genus):

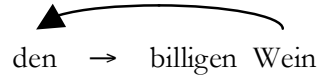

Regens ist der Kern, direktes Rektum der Kopf, indirektes Rektum das Adjektiv. Das Genus wird intern bestimmt, ist eine inhärente grammatische Eigenschaft des Substantivs. Primär ist der Kopf morphologisch markiert (den vs. die, das).

3. R3 (Typ Numerus):

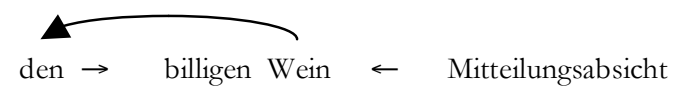

Regens ist der Kern, direktes Rektum der Kopf, indirektes Rektum das Adjektiv. Der Numerus wird extern bestimmt, aber nicht durch Rektion: Ein Sprecher wählt Numerus frei nach Mitteilungsabsicht. Primär ist der Kern morphologisch markiert (Wein vs. Weine).

Je nachdem, welche der drei $\mathrm{R}$ man zugrunde legt, kommt man zu verschiedenen Strukturbeschreibungen, die alle wohl begründbar sind. Wir sehen auch, dass die Festlegung des Kopfes von der Beschreibungsperspektive abhängt:

- Stellt man die abstrakte Kasusrelation in den Mittelpunkt, ist der Kopf das Determinans.

- Würde man dagegen die abstrakte Genus- oder Numerusrelation als zentral ansehen, wäre der Kopf das Substantiv. 
- Stellt man die konkrete morphologische Markierung der syntagmatischen Relationen in den Mittelpunkt, ist bezüglich Kasus und Genus das Determinans, bezüglich Numerus das Substantiv der Kopf.

- Würde man schließlich die Art der Bestimmung der jeweiligen Kategorie zugrunde legen, müsste man der internen Bestimmung (Genusrelation) höchste Kopfrelevanz zusprechen. Innerhalb der externen Bestimmungen würde dann die Bestimmung durch Rektion (Kasusrelation) höher rangieren als die freie Wahl nach Mitteilungsabsicht (Numerusrelation). Entsprechend hätte man keine Dichotomie (+/- Kopf), sondern eine Dreiteilung von maximaler über mittlere bis hin zu minimaler Köpfigkeit.

Wir haben drei mögliche Begründungen von Dependenz aufgeführt. Keine der drei definiert einen vollständigen Dependenzbaum im Sinne einer reinen DG: D1 führt zu Interdependenzen, D2 und D3 lassen Elemente isoliert, D3 ist ein Bündel von im Prinzip unabhängigen Relationen R1-R3. In der Praxis arbeiten Dependenzgrammatiker meist mit multirelationalen Dependenzbegriffen.

\subsection{Dependenz und Konstituenz}

Dependenz und Konstituenz wurden zunächst als konkurrierende Darstellungsmittel aufgefasst. Gaifman (1965) zeigte, dass die beiden Darstellungsmittel „schwach äquivalent“ sind: Die von einer projektiven Dependenzgrammatik generierten Ketten lassen sich auch von einer Phrasenstrukturgrammatik erzeugen. Auch wenn man die begrenzte Vergleichsbasis außer Acht lässt, bedeutet dies nicht, dass Dependenz und Konstituenz dasselbe sagen. Wir werden zeigen, dass das Gegenteil der Fall ist. Unsere Darstellung stützt sich auf Engel (1994: 23ff.) und Matthews (1981: 71ff., 2007: 112ff.) (s. auch Fischer 1997: 13ff., Uzonyi 2003).

Dependenz und Konstituenz basieren auf Konnexionen (geregeltem Miteinandervorkommen). Konstituenz stellt dar, dass durch Konnexionen verbundene Elemente ein größeres Ganzes bilden:

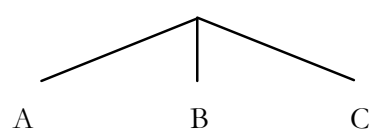

(Konstituenz)

Dependenz zeigt die Konnexionen direkt:

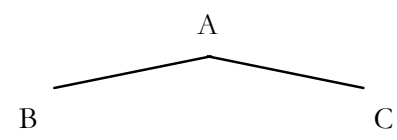

(Dependenz)

Die erste grundsätrliche Kritik der DG an Konstituenz ist, dass die Konnexionen nicht direkt gezeigt werden, sondern vermittelt über die Konstituente, deren Teil 
die Elemente sind, zwischen denen die Konnexionen bestehen. Obwohl die Beziehung zwischen den Elementen primär ist und Konstituenten sich erst aus dieser Beziehung ergeben, machen Konstituenzdarstellungen das Abgeleitete zum primären Datum.

Die Konstituenzdarstellung (48) ist komplexer als die Dependenzdarstellung (49). (48) behauptet Konnexionen zwischen allen drei Elementen, eine Dependenzdarstellung mit drei Elementen kann nur zwei Konnexionen zeigen. Die größere Komplexität von (48) ist nur dann ein Vorteil, wenn alle drei Konnexionen tatsächlich zutreffen. Generell ist es ein Problem für Konstituenzdarstellungen, dass sie zu starke Annahmen machen.

Konstituenzdarstellungen erlauben weitere Differenzierungen:

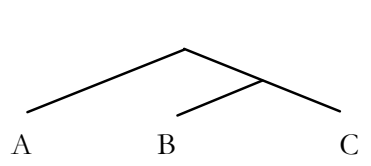

(Konstituenz)

In (50) wird behauptet, dass A mit der BC-Gruppierung eine Konnexion eingeht. Gruppierungen betreffende Konnexionen sind in einer reinen DG nur beschränkt darstellbar:

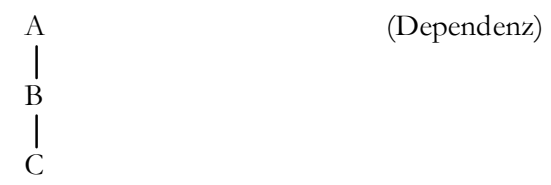

Hier bleibt es zwar bei zwei Konnexionen, aber C ist über Transitivität indirekt auch von A abhängig, BC bildet also mit dem Nucleus A einen Knoten. Problematisch ist allerdings, wenn $\mathrm{AB}$ eine Gruppierung bildet. Dies könnte etwa bei der Adjektivdeklination im Deutschen angenommen werden (s. der billige Wein), da sie sowohl kopf- als auch kerngesteuert ist (s. R1-R3 in 3.3). Hier muss man die Zusatzannahme machen, dass eine dependentielle Struktur selber als Nucleus eines Knotens auftreten kann (zur Begründung s. Lobin 1993): $(A \rightarrow B) \rightarrow C$.

Wir können die bisherige Diskussion so zusammenfassen, dass beim Vergleich der Leistung der Darstellungsmittel Dependenz und Konstituenz die Leistung in Relation zum Aufwand zu betrachten ist. Insbesondere müssen Zusatzannahmen und die Darstellung nicht-intendierter Struktur (starke Annahmen) berücksichtigt werden.

Die zweite grundsätzliche Kritik der DG an Konstituenz ist, dass sie keine natürliche Darstellung eines Kopfes erlaubt, während in der DG eine direkte Darstellung des Regens (Kopfes) erfolgt. Man vergleiche zwei übliche Konstituenzanalysen mit zwei üblichen Dependenzdarstellungen:

$$
\text { [[sehr alte] Bücher] } \quad \text { vs. } \quad \text { sehr } \leftarrow \text { alte } \leftarrow \text { Bücher }
$$




$$
\text { [kein [frisches Brot]] }
$$$$
\text { vs. } \quad \text { kein frisches } \leftarrow \text { Brot }
$$

Es lässt sich keine Anweisung formulieren, die aus den beiden Konstituenzdarstellungen die jeweiligen Köpfe ableitet. Köpfe müssen durch zusätzliche Beschreibungsmittel ausgezeichnet werden, etwa indem das X-bar-Schema und die Dependenzrelationen c-command und $\mathrm{m}$-command eingeführt werden und damit auch eine Konvention, nach der nur Köpfe Nicht-Phrasen sein dürfen, c- oder $\mathrm{m}$-kommandierte abhängige Elemente dagegen Phrasen sein müssen.

Aber auch Konstituenzdarstellungen enthalten Informationen, die sich nicht dependentiell nachzeichnen lassen: Es ist aus der Dependenzdarstellung (53) nicht ableitbar, dass die dependentielle Struktur von frisches Brot selber Nucleusqualitäten hat.

Wir können festhalten, dass Dependenz und Konstituenz nicht nur Konnexionen unterschiedlich erfassen, sondern auch unterschiedliche Aussagen machen: Der Dependenz ist das Konzept einer Gruppierung genauso fremd wie der Konstituenz das Konzept eines Regens. Deshalb sollten die beiden Prinzipien als komplementär betrachtet werden. Dependentielle Darstellungen werden gelegentlich punktuell konstitutionell erweitert. Die generative Grammatik hat wie erwähnt mit dem X-bar Schema und den Relationen c-command und m-command das Modell um Dependenzrelationen erweitert. Diese Entwicklungen haben die Kontroverse um das bessere Grundprinzip entschärft und deuten eine konvergente Entwicklung an (s. bereits Schmidt 1991).

\section{Quo vadis Grammatiktheorie?}

Auch wenn manche Handbücher und Einführungen (für wehrlose Anfänger) den Lesern suggerieren mögen, dass die allein selig machende grammatische Lehre bereits da sei, müssen wir festhalten, dass weder die DG noch andere Theorien das Prädikat beste Theorie für sich beanspruchen können. Wie Jacobs (2008) überzeugend zeigt, gibt es eine Reihe von zentralen, nicht marginalen grammatischen Phänomenen, die sich nur oder eher konstruktionistisch adäquat beschreiben lassen, und eine (andere) Reihe von ebenfalls zentralen grammatischen Phänomenen, die sich umgekehrt nur oder eher projektionistisch fassen lassen. Eine Konvergenz zwischen projektionistischen und konstruktionistischen Grammatikmodellen ist unbedingt erforderlich (s. auch 1.4 oben). Die Zukunft der DG - wie auch von anderen Theorien - hängt demnach in hohem Maße davon ab, ob sich im DG-Rahmen ein Theorieformat entwickeln lässt, das eine nicht durch ad-hoc- 
Stipulationen, sondern empirisch und methodologisch abgesicherte Integration von projektionistischen und konstruktionistischen Modellkomponenten enthält. ${ }^{14}$

In der VT hat die Integration von projektionistischen und konstruktionistischen Modellkomponenten bereits begonnen (Ágel 2003 und 2004, Coene/Willems 2006 und Willems/Coene 2006, Welke 2009a und 2009b). Dabei geht es vor allem um die Frage, wie sich ,Klassiker' der konstruktionsgrammatischen Theoriebildung wie Caused Motion-, Ditransitive-, Resultative- und Intransitive MotionKonstruktionen valenztheoretisch interpretieren und in die Theorie integrieren lassen. $^{15}$

\section{Literatur}

Ágel, Vilmos (1993): Ist die Dependenzgrammatik wirklich am Ende? Valenzrealisierungsebenen, Kongruenz, Subjekt und die Grenzen des syntaktischen Valenzmodells. In: ZGL 21, 20-70.

Ágel, Vilmos (2000): Valenztheorie. Tübingen: Narr (Narr Studienbücher).

Ágel, Vilmos (2001): Gegenwartsgrammatik und Sprachgeschichte. Methodologische Überlegungen am Beispiel der Serialisierung im Verbalkomplex. In: ZGL 29, 319-331.

Ágel, Vilmos (2003): Wort- und Ausdrucksvalenz(träger). In: Cornell, Alan/Fischer, Klaus/ Roe, F. Ian (Hg.): Valency in Practice/Valenz in der Praxis. Oxford et al.: Lang (German Linguistic and Cultural Studies 10), 17-36.

Ágel, Vilmos (2004): Phraseologismus als (valenz)syntaktischer Normalfall. In: Steyer, Kathrin (Hg.): Wortverbindungen - mehr oder weniger fest. Jahrbuch 2003 des Instituts für Deutsche Sprache. Berlin/New York: Walter de Gruyter, 65-86.

Ágel, Vilmos/Fischer, Klaus (2010): Dependency Grammar and Valency Theory. In: Heine, Bernd/Narrog, Heiko (Hg.): The Oxford Handbook of Linguistic Analysis. Oxford: Oxford University Press, 223-255.

Bianco, Maria T. (1996): Valenzlexikon deutsch-italienisch. 2 Bde. Heidelberg: Groos (Deutsch im Kontrast 17).

Bloomfield, Leonard (1933): Language. New York: Holt, Rinehart and Winston.

Bühler, Karl (1934/1982): Sprachtheorie. Die Darstellungsfunktion der Sprache. Stuttgart/ New York: Fischer (Uni-Taschenbücher 1159).

Chomsky, Noam (1970): Remarks on nominalization. In: Jacobs, Roderick A./Rosenbaum, Peter S. (Hg.): Readings in English Transformational Grammar Waltham, MA: Ginn, 184-221.

Coene, Ann (2006): Lexikalische Bedeutung, Valenz und Koerzion. Hildesheim et al.: Olms (Germanistische Linguistik Monographien 19).

14 Dasselbe gilt - mit umgekehrtem Vorzeichen - für die Konstruktionsgrammatik, die im Augenblick noch weniger mit dem Integrationsgedanken als vielmehr damit beschäftigt ist, den Konstruktionsbegriff auf alles Sprachliche auszudehnen und damit ihren ursprünglich weniger extensiven, aber fest umrissenen grammatischen Gegenstandsbereich durch eine Konstruktionsinflation zu zerstören.

15 Umgekehrt wartet man vergeblich darauf, dass ,Klassiker` der valenztheoretischen Theoriebildung wie etwa die E/A-Unterscheidung, Fakultativität/Weglassbarkeit, Varianz/Alternanz, Makro-/Mikrorealisierung konstruktionsgrammatisch interpretiert werden. 
Coene, Ann/Willems, Klaas (2006): Konstruktionelle Bedeutungen. Kritische Anmerkungen zu Adele Goldbergs konstruktionsgrammatischer Bedeutungstheorie. In: Sprachtheorie und germanistische Linguistik 16, 1-35.

Colleman, Timothy/Defrancq, Bart/Devos, Filip/Noël, Dirk (2004): The Contragram Dutch - French - English Contrastive Verb Valency Dictionary, Gent: University of Gent (http://www.contragram.ugent.be/cvvd.htm, Zugriff: 14.7.09).

Colliander, Peter (1999): Partikelvalenz im Deutschen. Eine prototypenlinguistische Studie über die Valenzverhältnisse. In: Zeitschrift für deutsche Sprache 27, 27-51.

Collins Dictionary of the English Language (1985). London/Glasgow: Collins.

Dik, Simon C. (1978): Functional Grammar. Amsterdam: North Holland/London: Academic Press.

Durrell, Martin (2002): Hammer's German Grammar and Usage. $4^{\text {th }}$ ed. London: Arnold.

Durrell, Martin (2010): Über den praktischen Nutzen der Valenztheorie im DaF-Unterricht. Eine kritische Bestandsaufnahme. In: Fischer, Klaus/Fobbe, Eilika/Schierholz, Stefan J. (Hg.): Valenz und Deutsch als Fremdsprache. Frankfurt a.M. [etc.]: Lang (Deutsche Sprachwissenschaft international 6),143-155.

Eisenberg, Peter (2006): Grundriss der deutschen Grammatik. Bd. 2: Der Satz. 3. Aufl. Stuttgart, Weimar: Metzler.

Emons, Rudolf (1974): Valenzen englischer Prädikatsverben. Tübingen: Niemeyer.

Engel, Ulrich (1988): Deutsche Grammatik. Vollständige Darstellung der deutschen Gegenwartssprache. Heidelberg: Groos.

Engel, Ulrich (1994): Syntax der deutschen Gegenwartssprache. 3., völlig neu bearb. Aufl. Berlin: Schmidt (Grundlagen der Germanistik 22).

Engel, Ulrich (1999): Deutsch-polnische kontrastive Grammatik. 2 Bde. Heidelberg: Groos.

Engel, Ulrich (2004): Deutsche Grammatik. Neubearbeitung. München: Iudicium.

Engel, Ulrich/Mrazović, Pavica (Hg.) (1986): Kontrastive Grammatik deutsch-serbokroatisch. 2 Bde. München: Sagner.

Engel, Ulrich/Isbăşescu, Mihai/Stănescu, Speranţa/Nicolae, Octavian (1993): Kontrastive Grammatik deutsch-rumänisch. Heidelberg: Groos.

Engelen, Bernhard (2003): Die Wortartenlehre bei Lucien Tesnière. In: Handbuch 2003, $100-108$.

Eroms, Hans-Werner (1985): Eine reine Dependenzgrammatik für das Deutsche. In: Deutsche Sprache 13, 306-326.

Eroms, Hans-Werner (2000): Syntax der deutschen Sprache. Berlin, New York: Walter de Gruyter (de Gruyter Studienbuch).

Eroms, Hans-Werner/Heringer, Hans Jürgen (2003): Dependenz und lineare Ordnung. In: Handbuch 2003, 247-263.

Feilke, Helmuth (1998): Idiomatische Prägung. In: Barz, Ingrid/Öhlschläger, Günther (Hg.): Zwischen Grammatik und Lexikon. Tübingen: Niemeyer (Linguistische Arbeiten 390), 69-80.

Feilke, Helmuth (2004): Kontext - Zeichen - Kompetenz. Wortverbindungen unter sprachtheoretischem Aspekt. In: Steyer, Kathrin (Hg.): Wortverbindungen - mehr oder weniger fest. Jahrbuch 2003 des Instituts für Deutsche Sprache. Berlin/New York: Walter de Gruyter, 41-64.

Fillmore, Charles J. (1968): The case for case. In: Bach, Emmon/Harms, Robert T. (Hg.): Universals in Linguistic Theory. New York: Holt, Rinehart \& Winston, 1-88.

Fillmore, Charles J. (1977): Scenes-and-frames semantics. In: Zampoli, Antonio (Hg.): Linguistic Structures Processing. Amsterdam/New York/Oxford: North-Holland, 55-81. 
Fischer, Klaus (1997): German-English Verb Valency. A Contrastive Analysis. Tübingen: Narr (Tübinger Beiträge zur Linguistik 422).

Fischer, Klaus (1999): Verb valency—an attempt at conceptual clarification. In: Web Journal of Modern Language Linguistics 4-5. (http://wjmll.ncl.ac.uk/issue04-05/fischer. htm, Zugriff: 15.7.09)

Fischer, Klaus (2001): Noch immer: Ergänzungen und Angaben. In: Sprachwissenschaft $26.3,239-268$.

Fischer, Klaus (2003): Verb, Aussage, Valenzdefinition und Valenzrealisierung: auf dem Weg zu einer typologisch adäquaten Valenztheorie. In: Willems, Klaas/Coene, Ann/Pottelberge, Jeroen Van (Hg.): Valenztheorie. Neuere Perspektiven. Akademia Press: Gent (Studia Germanica Gandensia 2003/2), 14-64.

Fischer, Klaus (2007a): Temporary ambiguity of German and English term complements. In: Herbst, Thomas/Götz-Votteler, Katrin (Hg.): Valency. Theoretical, Descriptive and Cognitive Issues. Berlin/New York: Mouton de Gruyter (Trends in Linguistics, Studies and Monographs 187), 229-252.

Fischer, Klaus (2007b): Komplexität und semantische Transparenz im Deutschen und Englischen. In: Sprachwissenschaft 32.4, 355-405.

Fobbe, Eilika (2010): Was von Valenz übrig bleibt. Die Rolle der Valenzgrammatik in Lehrwerken des Deutschen als Fremdsprache. In: Fischer, Klaus/Fobbe, Eilika/Schierholz, Stefan J. (Hg.): Valenz und Deutsch als Fremdsprache. Frankfurt a.M. [etc.]: Lang, 61-86.

Frege, Gottlob (1879): Begriffsschrift, eine der arithmetischen nachgebildete Formelsprache des reinen Denkens. Halle: Nebert.

Gaifman, Haim (1965): Dependency systems and phrase structure systems. In: Information and Control 8, 304-337.

Gansel; Christina (2003): Valenz und Kognition. In: Handbuch 2003, 422-444.

Givón, Talmy (1995): Functionalism and Grammar. Amsterdam/Philadelphia: Benjamins.

Greenberg, Joseph (1963): Some Universals of Grammar with Particular Reference to the Order of Meaningful Elements. In: Greenberg, Joseph (Hg.): Universals of Language. Cambridge: MIT Press, 73-113.

Groß, Thomas Michael (2003): The Valency of Non-Verbal Word Classes: the Adjective. In: Handbuch 2003, 835-842

Habermann, Mechthild (2007): Aspects of a diachronic valency syntax of German. In: Herbst, Thomas/Götz-Votteler, Katrin (Hg.): Valency. Theoretical, Descriptive and Cognitive Issues. Berlin/New York: Mouton de Gruyter (Trends in Linguistics, Studies and Monographs 187), 85-100.

Halliday, Michael A. K. (1994): An Introduction to Functional Grammar. Second Edition. London: Arnold.

Handbuch $2003=$ Dependenz und Valenz/Dependency and Valency. Ein internationales Handbuch der zeitgenössischen Forschung/An International Handbook of Contemporary Research. 1. Halbband. Hrsg. v. Ágel, Vilmos/Eichinger, Ludwig M./Eroms, Hans Werner/Hellwig, Peter/Heringer, Hans Jürgen/Lobin, Henning. Berlin/New York: Walter de Gruyter (Handbücher zur Sprach- und Kommunikationswissenschaft 25.1).

Handbuch 2006 = Dependenz und Valenz/Dependency and Valency. Ein internationales Handbuch der zeitgenössischen Forschung/An International Handbook of Contemporary Research. 2. Halbband. Hrsg. v. Ágel, Vilmos/Eichinger, Ludwig M./Eroms, Hans Werner/Hellwig, Peter/Heringer, Hans Jürgen/Lobin, Henning. Berlin/New York: Walter de Gruyter (Handbücher zur Sprach- und Kommunikationswissenschaft 25.2). 
Hawkins, John A. (1986): A Comparative Typology of English and German. Unifying the Contrasts. London, Sydney: Croom Helm.

Helbig, Gerhard (1992): Probleme der Valenz- und Kasustheorie. Tübingen: Niemeyer (Konzepte der Sprach- und Literaturwissenschaft 51).

Helbig, Gerhard/Schenkel, Wolfgang (1969): Wörterbuch zur Valenz und Distribution deutscher Verben. 8. Aufl. 1991. Tübingen: Niemeyer.

Herbst, Thomas (2007): Valency complements or valency patterns? In: Herbst, Thomas/ Götz-Votteler, Katrin (Hg.): Valency. Theoretical, Descriptive and Cognitive Issues. Berlin/New York: Mouton de Gruyter (Trends in Linguistics, Studies and Monographs 187), 15-35.

Herbst, Thomas/Heath, David/Roe, Ian F./Götz, Dieter (2004): A Valency Dictionary of English. Berlin/New York: Mouton de Gruyter.

Heringer, Hans Jürgen (1984): Neues von der Verbszene. In: Stickel, Gerhard (Hg.): Pragmatik in der Grammatik. Jahrbuch 1983 des Instituts für deutsche Sprache. Düsseldorf: Schwann (Sprache der Gegenwart 60), 34-64.

Heringer, Hans Jürgen (1985): The Verb and its Semantic Power: Association as a Basis for Valency Theory. In: Journal of Semantics 4, 79-99.

Heringer, Hans Jürgen (1996): Deutsche Syntax dependentiell. Tübingen: Stauffenburg (Stauffenburg Linguistik).

Heringer, Hans Jürgen (2006): Prinzipien des Valenzwandels. In: Handbuch 2006, 1447-1461.

Hölzner, Matthias (2007): Substantivvalenz. Korpusgestützte Untersuchungen zu Argumentrealisierungen deutscher Substantive. Tübingen: Niemeyer (RGL 274).

Huddleston, Rodney/Pullum, Geoffrey K. (2002): The Cambridge Grammar of the English Language. Cambridge: Cambridge University Press.

Hudson, Richard (2006): Language Networks. The New Word Grammar. Oxford: Oxford University Press.

Jackendoff, Ray (1977): X-bar-Syntax: A Study of Phrase Structure. Linguistic Inquiry Monograph 2. Cambridge, MA: MIT Press.

Jacobs, Joachim (1994): Kontra Valenz. Trier: Wissenschaftlicher Verlag (Fokus 12).

Jacobs, Joachim (2003): Die Problematik der Valenzebenen. In: Handbuch 2003, 378-399.

Jacobs, Joachim (2008): Wozu Konstruktionen? In: Linguistische Berichte 213, 3-44.

Janich, Peter (2009): Kein neues Menschenbild. Zur Sprache der Hirnforschung. Frankfurt am Main: Suhrkamp (edition unseld 21).

Katz, Jerrold J./Fodor, Jerry A. (1963). The structure of a Semantic Theory. In: Language $39,170-210$.

Korhonen, Jarmo (2006): Valenzwandel am Beispiel des Deutschen. In: Handbuch 2006, 1462-1474.

László, Sarolta (1988): Mikroebene. In: Mrazović, Pavica/Teubert, Wolfgang (Hg.): Valenzen im Kontrast. Ulrich Engel zum 60. Geburtstag. Heidelberg: Groos, 218-233.

Leiss, Elisabeth (2002): Die Wortart ,Verb‘. In: Cruise, D. Alan/Hundsnurscher, Frans/Job, Michael/Lutzeier, Peter (Hg.): Lexikologie. Ein internationales Handbuch zur Natur und Struktur von Wörtern und Wortschätzen. Bd. 1. Berlin/New York: Walter de Gruyter (Handbücher zur Sprach- und Kommunikationswissenschaft 15.1), 605-616.

Leiss, Elisabeth (2003): Empirische Argumente für Dependenz. In: Handbuch 2003, 311324.

Lobin, Henning (1993): Koordinationssyntax als prozedurales Phänomen. Tübingen: Narr (Studien zur deutschen Grammatik 46).

Matthews, Peter H. (1981): Syntax. Cambridge: Cambridge University Press. 
Matthews, Peter H. (2007): Syntactic Relations. A Critical Survey. Cambridge: Cambridge University Press (Cambridge Studies in Linguistics 114).

Meiner, Johann Werner (1781/1971): Versuch einer an der menschlichen Sprache abgebildeten Vernuftlehre oder Philosophie und allgemeine Sprachlehre. Stuttgart-Bad Cannstadt: Frommann (Grammatica Universalis 6).

Mel'čuk, Igor (1988): Dependency Syntax: Theory and Practice. Albany, NY: State University of New York Press.

Mel'čuk, Igor (2003): Levels of Dependency in Linguistic Description: Concepts and Problems. In: Handbuch 2003, 188-229.

Nichols, Johanna (1986): Head-marking and dependent-marking grammar. In: Language 62, 56-119.

Owens, Jonathan (2003): Valency-like Concepts in the Arabic Grammatical Tradition. In: Handbuch 2003, 26-32.

Pasierbsky, Fritz (1981): Sprachtypologische Aspekte der Valenztheorie unter besonderer Berücksichtigung des Deutschen. In: Zeitschrift für Phonetik, Sprachwissenschaft und Kommunikationsforschung 34, 160-177.

Percival, Keith (1990): Reflections on the History of Dependency Notions in Linguistics. In: Historiographia Linguistica XVII, 29-47.

Plank, Frans (1984): Verbs and objects in semantic agreement: minor differences between languages that might suggest a major one. In: Journal of Semantics 3, 305-360.

Quirk, Randolph/Greenbaum, Sidney/Leech, Geoffrey/Svartvik, Jan (1985): A Comprehensive Grammar of the English Language. London/New York: Longman.

Reichmann, Oskar (1988): Zur Vertikalisierung des Varietätenspektrums in der jüngeren Sprachgeschichte des Deutschen. In: Munske, Horst Haider/Polenz, Peter von/ Reichmann, Oskar/Hildebrandt, Reiner (Hg.): Deutscher Wortschatz. Lexikologische Studien. Festschrift für Ludwig Erich Schmitt von seinen Marburger Schülern. Berlin/New York: Walter de Gruyter, 151-180.

Schmidt, Jürgen Erich (1991): Konvergenzen zwischen neueren Grammatiktheorien und Deskriptionsgrammatiken? Zum Verhältnis von Konstituenz, Rektion (Government), Valenz und Dependenz. In: Feldbusch, Elisabeth/Pogarell, Reiner/Weiß, Cornelia (Hg.): Neue Fragen der Linguistik, Akten des 25. Linguistischen Kolloquiums, Paderborn 1990. Bd. 1: Bestand und Entwicklung. Tübingen: Niemeyer (Linguistische Arbeiten 270), 211-218.

Seidel, Kurt Otto (2003): Valenzverwandte Ansätze in der Antike. In: Handbuch 2003, 14 20.

Seppänen, Lauri (2003): Mit der Valenz verwandte Begriffe im Mittelalter: ein Überblick. In: Handbuch 2003, 20-26.

Somers, Harold L. (1987): Valency and Case in Computational Linguistics. Edinburgh: Edinburgh University Press (Edinburgh Information Technology Series 3).

Starosta, Stanley (1988): The Case for Lexicase. An Outline of Lexicase Grammatical Theory. London: Pinter.

Storrer, Angelika (1992): Verbvalenz. Theoretische und methodische Grundlagen ihrer Beschreibung in Grammatikographie und Lexikographie. Tübingen: Niemeyer (Reihe Germanistische Linguistik 126).

Storrer, Angelika (2003): Ergänzungen und Angaben. In: Handbuch 2003, 764-780.

Tesnière, Lucien (1953): Esquisse d'une syntaxe structurale. Paris: Klincksieck.

Tesnière, Lucien (1976): Éléments de syntaxe structurale. 2e édition revue et corrigée, $3 e$ tirage. Paris: Klincksieck. 
Tesnière, Lucien (1980): Grundzüge der strukturalen Syntax. Hg. und übers. von Ulrich Engel. Stuttgart: Klett-Cotta.

Teubert, Wolfgang (2003): Die Valenz nichtverbaler Wortarten: das Substantiv. In: Handbuch 2003, 820-835.

Uzonyi, Pál (2003): Dependenzstruktur und Konstituenzstruktur. In: Handbuch 2003, 230-247.

VALBU $=$ Schumacher, Helmut/Kubczak, Jacqueline/Schmidt, Renate/Ruiter, Vera de (2004). VALBU - Valenzwörterbuch deutscher Verben. Tübingen: Narr.

Weber, Heinz J. (1997): Dependenzgrammatik. Ein interaktives Arbeitsbuch. 2., überarb. Aufl. Tübingen: Narr (Narr Studienbücher).

Welke, Klaus M. (1988): Einführung in die Valenz- und Kasustheorie. Leipzig: VEB Bibliographisches Institut.

Welke, Klaus M. (1995): Dependenz, Valenz und Konstituenz. In: Eichinger, Ludwig M./ Eroms, Hans-Werner (Hg.): Dependenz und Valenz. Hamburg: Buske (Beiträge zur germanistischen Sprachwissenschaft 10), 163-175.

Welke, Klaus M. (2005): Deutsche Syntax funktional. Perspektiviertheit syntaktischer Strukturen. 2. Aufl. Tübingen: Stauffenburg (Stauffenburg Linguistik 22).

Welke, Klaus (2009a): Valenztheorie und Konstruktionsgrammatik. In: ZGL 37, 81-124.

Welke, Klaus (2009b): Konstruktionsvererbung, Valenzvererbung und die Reichweite von Konstruktionen. In: ZGL 37, 514-543.

Willems, Klaas/Coene, Ann (2006): Satzmuster und die Konstruktionalität der Verbbedeutung. Überlegungen zum Verhältnis von Konstruktionsgrammatik und Valenztheorie. In: Sprachwissenschaft 31, 237-272.

Zifonun, Gisela/Hoffmann, Ludger/Strecker, Bruno (1997): Grammatik der deutschen Sprache. 3 Bde. Berlin/New York: Walter de Gruyter.

Adressen der Verfasser:

Prof. Dr. Vilmos Agel, FB 02 - Germanistik, Universität Kassel, Kurt-Wolters-Straße 5, D-34125 Kassel.

E-Mail: ágel@uni-kassel.de

Dr. Klaus Fischer, London Metropolitan University, Faculty of Humanities, Arts, Languages and Education, 166-220 Holloway Road, London N7 8DB, Großbritannien.

E-Mail: k.fischer@londonmet.ac.uk 